\title{
SPECTRAL ASYMPTOTICS FOR TWO-DIMENSIONAL SCHRÖDINGER OPERATORS WITH STRONG MAGNETIC FIELDS*
}

\author{
ANH TUAN DUONG ${ }^{\dagger}$
}

Abstract. In this paper we study the perturbed quadratic Hamiltonian in two-dimensional case, $P(b, \omega)=\left(D_{x}-b y\right)^{2}+D_{y}^{2}+\omega^{2} x^{2}-\sqrt{b^{2}+\omega^{2}}+V(x, y)$. Here, $b$ is the strong constant magnetic field, $\omega \neq 0$ is a fixed constant, and the potential $V$ vanishes at infinity. For $f \in C_{0}^{\infty}((-\infty, 0) ; \mathbb{R})$ and $b$ large enough, we give a full asymptotic expansion in powers of $b^{-1}$ of the trace of $f(P(b, \omega))$. Moreover, we also obtain a Weyl formula with optimal remainder estimate of the counting function of eigenvalues of $P(b, \omega)$ as $b \rightarrow \infty$.

Key words. Schrödinger operators, strong magnetic fields, asymptotic trace formula, eigenvalues distribution.

AMS subject classifications. 81Q10, 35J10, 35P20, 35C20, 47F05.

1. Introduction. We consider the two-dimensional Schrödinger operator with constant magnetic field

$$
\begin{aligned}
P(b, \omega) & =P_{0}(b, \omega)+V(x, y) \\
& =\left(D_{x}-b y\right)^{2}+D_{y}^{2}+\omega^{2} x^{2}-\sqrt{b^{2}+\omega^{2}}+V(x, y), \quad(x, y) \in \mathbb{R}^{2},
\end{aligned}
$$

where $D_{\nu}=\frac{1}{i} \partial_{\nu}, b$ is the strong constant magnetic field and $\omega \neq 0$ is a fixed constant.

Throughout this paper, we always assume that the electric potential $V$ satisfies the following hypothesis:

(H) The potential $V$ is a real-valued smooth function, bounded with all its derivatives, and tends to zero at infinity, i.e., $\forall \beta, \gamma \in \mathbb{N}$

$$
\left|\partial_{x}^{\beta} \partial_{y}^{\gamma} V(x, y)\right| \leq C_{\beta, \gamma}, \forall(x, y) \in \mathbb{R}^{2} .
$$

It is well known that the operator $P(b, \omega)$ is essentially self-adjoint on $C_{0}^{\infty}\left(\mathbb{R}^{2}\right)$ (see $[14,21,22])$. For $V \equiv 0$, it was shown that the spectrum of the unperturbed operator $P_{0}(b, \omega)$ is absolutely continuous and equal to the interval $[0, \infty)$ (see $[22]$ ). Since $V$ tends to zero at infinity, the operator $V\left(P_{0}(b, \omega)+i\right)^{-1}$ is compact (see [1]). According to the Weyl theorem (see [27]), the operators $P(b, \omega)$ and $P_{0}(b, \omega)$ have the same essential spectrum, i.e., $\sigma_{\text {ess }}(P(b, \omega))=\sigma_{\text {ess }}\left(P_{0}(b, \omega)\right)=[0, \infty)$. Thus the spectrum of $P(b, \omega)$ in the interval $(-\infty, 0)$ is discrete.

Recently a substantial progress has been made in the analysis of the magnetic Schrödinger operators with long-range perturbations going to 0 as $|x| \rightarrow+\infty$ and the works around the trace formulae have generated many results on the distribution of eigenvalues near Landau levels and Weyl's formula with sharp remainder estimate of the counting function of eigenvalues (see [1, 5, 8, 9, 11, 14, 16, 18, 19, 23, 24, 25, 26, 29] and the references given there).

To our best knowledge, there are only a few works concerning the model (1.1) (see $[14,21,22])$. In [22], the authors studied a quadratic Hamiltonian without perturbation by using the theory of metapletic representations. In [14], the authors investigated the absolutely continuous spectrum of $P(b, \omega)$. By applying the Mourre

\footnotetext{
*Received November 15, 2011; accepted for publication July 26, 2012.

†LAGA, (UMR CNRS 7539), Univ. Paris 13, F-93430 Villetaneuse, France (duongat@math.univparis13.fr).
} 
theory, they proved that a part of the absolutely continuous spectrum of $P(b, \omega)$ persists. On the other hand, we can consider the model (1.1) as the quantum hall system Hamiltonian with the unbounded edge potential $W(x)=\omega^{2} x^{2}$ (see $\left.[4,6,20]\right)$.

In this work, we give a complete asymptotic expansion in powers of $b^{-1}$ of the trace of the operators $f(P(b, \omega))$ and $f(P(b, \omega)) \mathcal{F}_{\frac{1}{\alpha}}^{-1} \theta(\lambda-P(b, \omega))$, where $f \in$ $C_{0}^{\infty}((-\infty, 0) ; \mathbb{R}), \theta \in C_{0}^{\infty}\left(\left(-\frac{1}{C}, \frac{1}{C}\right) ; \mathbb{R}\right)$ with $C$ large enough. Here $\alpha:=\sqrt{b^{2}+\omega^{2}}$ and $\mathcal{F}_{\frac{1}{\alpha}}^{-1} \theta(x)=\frac{\alpha}{2 \pi} \int_{\mathbb{R}} e^{i \alpha t x} \theta(t) d t$. The proof is based on the effective Hamiltonian method and the stationary techniques developed by M. Dimassi [7] (see also M. Dimassi-J. Sjöstrand [12]). More precisely, we reduce the spectral study of the operator $P(b, \omega)$ near a fixed energy $z$ to the study of a $\frac{1}{\alpha}-$ pseudodifferential operator $E_{-+}(z)$ called the effective Hamiltonian. Then we apply to the operator $E_{-+}(z)$ the time independent approach. Furthermore, thanks to a Tauberian theorem (see $[8,28]$ ), we deduce Weyl's formula with optimal remainder estimate of the counting function of isolated eigenvalues of $P(b, \omega)$ in $(-\infty, \lambda]$, where $\lambda<0$ is a fixed constant.

The paper is organized as follows. In Section 2 we recall some notations and definitions for symbols and pseudodifferential operators. Our main results are announced in Section 3. In Subsection 4.1, we establish and study the effective Hamiltonian. Then we prove some trace formulae in Subsection 4.2. The proofs of our main results are given in Section 5 and Section 6. Finally, we construct some linear canonical transformations in Appendix A.

2. Notations and definitions. In this paper, we will use the notations in $[12$, 13] for symbols and $h$ - pseudodifferential operators. Here $h$ is the semiclassical parameter.

Definition 2.1. A function $m: \mathbb{R}^{2 n} \rightarrow(0, \infty)$ is called an order function if there exist constants $C, N$ such that

$$
m(w) \leq C\langle\tilde{w}-w\rangle^{N} m(\tilde{w}),
$$

for all $\tilde{w}, w \in \mathbb{R}^{2 n}$.

Definition 2.2. Let $m$ be an order function on $\mathbb{R}^{2 n}$. For $k \in \mathbb{R}, 0 \leq l<\frac{1}{2}$, we define the class of symbols:

$$
S_{l}^{k}\left(m, \mathbb{R}^{2 n}\right):=\left\{a \in C^{\infty}\left(\mathbb{R}^{2 n}\right) \mid \forall \beta \in \mathbb{N}^{2 n}, \exists C_{\beta}>0 \text { s.t. }\left|\partial^{\beta} a\right| \leq C_{\beta} h^{-k-l|\beta|} m\right\} .
$$

If $m=1$ (resp. $l=0)$, we write $S_{l}^{k}\left(\mathbb{R}^{2 n}\right):=S_{l}^{k}\left(1, \mathbb{R}^{2 n}\right)\left(\right.$ resp. $S^{k}\left(m, \mathbb{R}^{2 n}\right):=$ $\left.S_{0}^{k}\left(m, \mathbb{R}^{2 n}\right)\right)$.

Definition 2.3. Let $a(. ; h) \in S_{l}^{k_{0}}\left(m, \mathbb{R}^{2 n}\right)$ depend on $h$. We say that $a(. ; h)$ admits an asymptotic expansion in powers of $h$, if there exists a sequence of symbols of $S_{l}^{k_{0}}\left(m, \mathbb{R}^{2 n}\right),\left(a_{j}\right)_{j \in \mathbb{N}}$, such that for any $N \in \mathbb{N}$, and for any $\beta \in \mathbb{N}^{2 n}$, there exists $C_{N, \beta}>0$, s.t.

$$
\left|\partial^{\beta}\left(a(. ; h)-\sum_{j=0}^{N} h^{j} a_{j}\right)\right| \leq C_{N, \beta} h^{N+1-k_{0}-l|\beta|} m .
$$

We write $a(. ; h) \sim \sum_{j=0}^{\infty} h^{j} a_{j}$ in $S_{l}^{k_{0}}\left(m, \mathbb{R}^{2 n}\right)$. 
Definition 2.4. (see [2]) We denote by $S^{0}\left(\mathbb{R}^{2 n} ; \mathcal{L}\left(L^{2}\left(\mathbb{R}^{n}\right)\right)\right)$ the set of operatorvalued functions $a \in C^{\infty}\left(\mathbb{R}^{2 n} ; \mathcal{L}\left(L^{2}\left(\mathbb{R}^{n}\right)\right)\right)$ satisfying:

For $\beta \in \mathbb{N}^{2 n}$, there exists $C_{\beta}>0$ such that

$$
\left\|\partial^{\beta} a(x, \xi)\right\|_{\mathcal{L}\left(L^{2}\left(\mathbb{R}^{n}\right)\right)} \leq C_{\beta}, \forall(x, \xi) \in \mathbb{R}^{2 n} .
$$

We will use the standard Weyl quantization of symbols. More precisely, if $a(x, \xi)$, $(x, \xi) \in \mathbb{R}^{2 n}$, is a symbol in $S_{l}^{k}\left(m, \mathbb{R}^{2 n}\right)\left(\operatorname{resp} . S^{0}\left(\mathbb{R}^{2 n} ; \mathcal{L}\left(L^{2}\left(\mathbb{R}^{n}\right)\right)\right)\right)$, then $a^{w}\left(x, h D_{x}\right)$ is the operator defined by

$$
a^{w}\left(x, h D_{x}\right) u(x):=\frac{1}{(2 \pi h)^{n}} \int_{\mathbb{R}^{n}} \int_{\mathbb{R}^{n}} e^{\frac{i}{h}\langle x-y, \xi\rangle} a\left(\frac{x+y}{2}, \xi\right) u(y) d y d \xi,
$$

for $u \in \mathcal{S}\left(\mathbb{R}^{n}\right)$ (resp. $u \in \mathcal{S}\left(\mathbb{R}^{n} ; L^{2}\left(\mathbb{R}^{n}\right)\right)$ ). Sometimes, we write $O p_{h}^{w}(a)$ instead of $a^{w}\left(x, h D_{x}\right)$. For the theory of $h$ - pseudodifferential operators with operator-valued symbols, we refer to $[2,3,15]$.

Let $g(\alpha)$ be a function depending on a large parameter $\alpha$. We say that $g$ has a complete asymptotic expansion in powers of $\alpha^{-1}$, and we write

$$
g(\alpha) \sim \sum_{j=0}^{\infty} c_{j} \alpha^{-j}, \text { as } \alpha \rightarrow \infty
$$

if and only if, for all $N \in \mathbb{N}$,

$$
\lim _{\alpha \rightarrow \infty}\left[g(\alpha)-\sum_{j=0}^{N} c_{j} \alpha^{-j}\right] \alpha^{N}=0
$$

We write $g(\alpha)=\mathcal{O}\left(\alpha^{-\infty}\right)$ if for all $N \in \mathbb{N}, g(\alpha)=\mathcal{O}_{N}\left(\alpha^{-N}\right)$.

3. Main results. In this section, we state our main results:

THEOREM 3.1. Let $f \in C_{0}^{\infty}((-\infty, 0) ; \mathbb{R})$ and assume that $V$ satisfies the assumption $(\mathbf{H})$. Then, the following full expansion holds:

$$
\operatorname{tr}(f(P(b, \omega))) \sim b \sum_{j=0}^{\infty} B_{j}(f) b^{-j}, \text { as } b \rightarrow \infty
$$

Moreover,

$$
\begin{aligned}
& B_{0}(f)=\frac{1}{2 \pi \omega} \iint f\left(x^{2}+V\left(\frac{x}{\omega}, y\right)\right) d x d y \\
& B_{1}(f)=\frac{1}{8 \pi \omega} \iint\left[\partial_{x}^{2} V\left(\frac{x}{\omega}, y\right)+\partial_{y}^{2} V\left(\frac{x}{\omega}, y\right)\right] f^{\prime}\left(x^{2}+V\left(\frac{x}{\omega}, y\right)\right) d x d y
\end{aligned}
$$

ThEOREM 3.2. Fix $\lambda<0$. In addition to the assumption $(\mathbf{H})$ suppose that $\nabla_{x, y}\left(x^{2}+V\left(\frac{x}{\omega}, \omega y\right)\right) \neq 0$ on the surface

$$
\left\{(x, y) \in \mathbb{R}^{2} \mid x^{2}+V\left(\frac{x}{\omega}, \omega y\right)=\lambda\right\}
$$


Then, there exist $\sigma>0$ small enough and $C>0$ large enough such that, for $f \in$ $C_{0}^{\infty}((\lambda-\sigma, \lambda+\sigma) ; \mathbb{R})$ and $\theta \in C_{0}^{\infty}\left(\left(-\frac{1}{C}, \frac{1}{C}\right) ; \mathbb{R}\right)$, there exist functions $C_{j}(\tau) \in C^{\infty}(\mathbb{R})$, $\forall M, N \in \mathbb{N}$, we have

$$
\operatorname{tr}\left(f(P(b, \omega)) \mathcal{F}_{\frac{1}{\alpha}}^{-1} \theta(\tau-P(b, \omega))\right)=b\left(\sum_{j=0}^{M} C_{j}(\tau) b^{-j}+\mathcal{O}\left(\frac{b^{-M-1}}{\langle\tau\rangle^{N}}\right)\right) \text {, as } b \rightarrow \infty
$$

uniformly in $\tau \in \mathbb{R}$. Here $\langle\tau\rangle=\left(1+\tau^{2}\right)^{\frac{1}{2}}$,

$$
C_{0}(\tau)=\frac{1}{2 \pi} f(\tau) \theta(0) \int_{\left\{(x, y) \in \mathbb{R}^{2} \mid x^{2}+V\left(\frac{x}{\omega}, \omega y\right)=\lambda\right\}} \frac{d S_{\tau}}{\left|\nabla_{x, y}\left(x^{2}+V\left(\frac{x}{\omega}, \omega y\right)\right)\right|},
$$

and $S_{\tau}$ is the surface measure on $\left\{(x, y) \in \mathbb{R}^{2} \mid x^{2}+V\left(\frac{x}{\omega}, \omega y\right)=\lambda\right\}$.

TheOREM 3.3. Fix $\lambda<0$, and let $N_{\alpha}(\lambda)$ be the number of eigenvalues of $P(b, \omega)$ in $(-\infty, \lambda]$ counted with their multiplicities. Under the assumptions of Theorem 3.2, we have

$$
N_{\alpha}(\lambda)=b\left(M_{0}+\mathcal{O}\left(b^{-1}\right)\right), \text { as } b \rightarrow \infty
$$

where

$$
M_{0}=\frac{1}{2 \pi \omega} \iint_{\left\{(x, y) \in \mathbb{R}^{2} \mid x^{2}+V\left(\frac{x}{\omega}, y\right) \leq \lambda\right\}} d x d y
$$

REMARK 3.4. Since $\alpha=\sqrt{b^{2}+\omega^{2}}$, and $\omega \neq 0$ is fixed, it follows that

$$
\alpha^{-1} \sim \sum_{j=1}^{\infty} c_{j}(\omega) b^{-j} \text { and } b^{-1} \sim \sum_{j=1}^{\infty} d_{j}(\omega) \alpha^{-j}
$$

Thus, we only need to prove that the left hand sides of (3.1), (3.4) and (3.6) have asymptotic expansions in powers of $\alpha^{-1}$.

Outline of the proof. Using linear changes of variables, we prove in Appendix A that the operator $P(b, \omega)$ is unitarily equivalent to the operator $\tilde{P}(\alpha):=\tilde{P}_{0}(\alpha)+$ $V^{w}(\alpha)$, where $\tilde{P}_{0}(\alpha):=\alpha\left(D_{y}^{2}+y^{2}\right)+x^{2}-\alpha$ and $V^{w}(\alpha):=V^{w}\left(\frac{1}{\sqrt{\alpha}} D_{y}+\frac{b}{\alpha \omega} x, \frac{\omega}{\alpha} D_{x}+\right.$ $\left.\frac{b \sqrt{\alpha}}{\alpha^{2}} y\right)$.

By constructing a suitable Grushin problem (see Lemma 4.3) we reduce the spectral study of $(\tilde{P}(\alpha)-z)$ for $z \in(\underline{a}, \bar{a}) \subset(-\infty, 0)$ to the study of a $\frac{1}{\alpha}-$ pseudodifferential operator $E_{+-}(z)$ called effective Hamiltonian. Here we notice that, in our case $(\omega \neq 0)$, the effective Hamiltonian is no longer a bounded operator from $L^{2}(\mathbb{R})$ to $L^{2}(\mathbb{R})$, because of the presence of the edge potential $W(x)=\omega^{2} x^{2}$ (see Lemma 4.3). This is the main difference from the work of M. Dimassi [8] where $\omega$ is assumed to be equal to zero. To overcome this difficulty, we show that if $f \in C_{0}^{\infty}((-\infty, 0)$; $\mathbb{R})$, we have $\operatorname{tr}(f(\tilde{P}(\alpha)))=\operatorname{tr}\left(f(\tilde{P}(\alpha)) \chi^{w}\right)+\mathcal{O}\left(\alpha^{-\infty}\right)$ for some compactly supported function $\chi$ (see formula (4.47)). Thus, by using the Helffer-Sjöstrand formula, we prove that (see formula (4.47))

$$
\operatorname{tr}\left(f(\tilde{P}(\alpha)) \chi^{w}\right)=\operatorname{tr}\left(-\frac{1}{\pi} \int \bar{\partial}_{z} \tilde{f}(z) E_{-+}(z)^{-1} \partial_{z} E_{-+}(z) \chi^{w} L(d z)\right) .
$$


Here, $\tilde{f} \in C_{0}^{\infty}(\mathbb{C})$ is an almost analytic extension of $f$. Then, we only need to study $E_{-+}(z)$ in the right hand side of (3.8) on the support of $\chi$. Next, by applying the $\frac{1}{\alpha}-$ pseudodifferential calculus, modulo $\mathcal{O}\left(\alpha^{-\infty}\right)$ we can replace $E_{-+}(z)$ in $(3.8)$ by a bounded operator $\tilde{E}(z)$ (see Proposition 4.7) such that

$$
\operatorname{tr}\left(f(\tilde{P}(\alpha)) \chi^{w}\right)=\operatorname{tr}\left(-\frac{1}{\pi} \int \bar{\partial}_{z} \tilde{f}(z) \tilde{E}(z)^{-1} \partial_{z} \tilde{E}(z) \chi^{w} L(d z)\right)+\mathcal{O}\left(\alpha^{-\infty}\right) .
$$

Moreover, we show that $\tilde{E}(z)$ has a complete asymptotic expansion in powers of $\frac{1}{\alpha}$ (see Proposition 4.5). Then by applying the time independent method used by M. Dimassi [7] (see also [12]), we prove Theorem 3.1 and Theorem 3.2.

By combining Theorems 3.1 and 3.2 with Tauberian arguments, we obtain the proof of Theorem 3.3.

4. The effective Hamiltonian and trace formulae. In this section, we construct the effective Hamiltonian $E_{-+}(z)$ and we give a trace formula linking the operators $\tilde{P}(\alpha)$ and $E_{-+}(z)$. Without loss of generality we may assume that $\omega=1$.

4.1. The effective Hamiltonian. Let $g$ be a positive function satisfying $g(\alpha) \rightarrow \infty$ as $\alpha \rightarrow \infty$. Set

$$
\Omega_{\alpha}:=\{z \in \mathbb{C} \mid \operatorname{Re} z \leq 2 \alpha-g(\alpha)\} .
$$

We denote by $\phi(y):=\pi^{-\frac{1}{4}} e^{-\frac{y^{2}}{2}}$ the normalized eigenfunction of the one-dimensional harmonic operator corresponding to the ground state energy $E=1$ (i.e., $\left(D_{y}^{2}+\right.$ $\left.y^{2}\right) \phi(y)=\phi(y)$ and $\left.\|\phi\|_{L^{2}(\mathbb{R})}=1\right)$. The following lemma is well known:

LEMmA 4.1. The following statements hold:

i) $\phi$ is an even function.

ii) $\left\langle D_{y}^{k} \phi(y), y^{l} \phi(y)\right\rangle=0$, when $k+l$ is an odd number.

iii) $\left\|\phi^{\prime}(y)\right\|_{L^{2}(\mathbb{R})}^{2}=\|y \phi(y)\|_{L^{2}(\mathbb{R})}^{2}=\frac{1}{2}$.

We now consider the following operators

$$
\begin{array}{cc}
R_{-}: L^{2}(\mathbb{R}) \rightarrow L^{2}\left(\mathbb{R}^{2}\right), & R_{+}: L^{2}\left(\mathbb{R}^{2}\right) \rightarrow L^{2}(\mathbb{R}), \\
u(x) \mapsto u(x) \phi(y) & v(x, y) \mapsto \int v(x, y) \phi(y) d y \\
\Pi:=R_{-} R_{+} \quad: L^{2}\left(\mathbb{R}^{2}\right) \rightarrow L^{2}\left(\mathbb{R}^{2}\right), \\
v(x, y) \mapsto \int v(x, t) \phi(t) d t \phi(y) .
\end{array}
$$

From the definition of $R_{-}$and $R_{+}$, we have

$$
R_{+} R_{-} u(x)=R_{+}(u(x) \phi(y))=\int u(x) \phi(y) \phi(y) d y=u(x) .
$$

LEMmA 4.2. There exists $\alpha_{0}>0$ large enough such that, for $\alpha \geq \alpha_{0}$, and $z \in \Omega_{\alpha}$, the operator

$$
(I-\Pi) \tilde{P}(\alpha)(I-\Pi)-z:(I-\Pi) L^{2}\left(\mathbb{R}^{2}\right) \rightarrow(I-\Pi) L^{2}\left(\mathbb{R}^{2}\right)
$$


is invertible. In addition, there exists $C>0$ such that

$$
\|R(z)\| \leq \frac{C}{g(\alpha)} \text { uniformly in } z \in \Omega_{\alpha}
$$

where $R(z):=((I-\Pi) \tilde{P}(\alpha)(I-\Pi)-z)^{-1}(I-\Pi): L^{2}\left(\mathbb{R}^{2}\right) \rightarrow L^{2}\left(\mathbb{R}^{2}\right)$.

Proof. In the following, we denote by $\sigma(A)$ (resp. $\rho(A)$ ) the spectrum (resp. resolvent set) of the operator $A$. It is clear that $\sigma\left((I-\Pi) \tilde{P}_{0}(\alpha)(I-\Pi)\right)=[2 \alpha,+\infty)$ (see [17, Proposition 6.9]). Hence, for $z \in \Omega_{\alpha}$, we have

$(4.3) \operatorname{dist}\left(z, \sigma\left((I-\Pi) \tilde{P}_{0}(\alpha)(I-\Pi)\right)\right)\|(I-\Pi) u\| \leq\left\|\left[(I-\Pi) \tilde{P}_{0}(\alpha)(I-\Pi)-z\right](I-\Pi) u\right\|$.

On the other hand, for $z \in \Omega_{\alpha}$, one has $g(\alpha) \leq \operatorname{dist}\left(z, \sigma\left((I-\Pi) \tilde{P}_{0}(\alpha)(I-\Pi)\right)\right)$. Thus,

$$
g(\alpha)\|(I-\Pi) u\| \leq\left\|\left[(I-\Pi) \tilde{P}_{0}(\alpha)(I-\Pi)-z\right](I-\Pi) u\right\| .
$$

Moreover, since $V$ is bounded simultaneously with all its derivatives, it follows from the Calderón-Vaillancourt theorem (see [12, Theorem 7.11]) that there exists $C_{1}>0$ such that

$$
\left\|V^{w}(\alpha)(I-\Pi) u\right\| \leq C_{1}\|(I-\Pi) u\| .
$$

Combining this with (4.4), we have

$$
\left(g(\alpha)-C_{1}\right)\|(I-\Pi) u\| \leq\|[(I-\Pi) \tilde{P}(\alpha)(I-\Pi)-z](I-\Pi) u\|
$$

uniformly in $z \in \Omega_{\alpha}$, which yields (4.2). We recall that $\lim _{\alpha \rightarrow \infty} g(\alpha)=+\infty$. $\square$ Next, we construct a Grushin problem for the perturbed Hamiltonian:

$$
\mathcal{P}(z)=\left(\begin{array}{cc}
\tilde{P}(\alpha)-z & R_{-} \\
R_{+} & 0
\end{array}\right): \mathcal{D} \times L^{2}(\mathbb{R}) \rightarrow L^{2}\left(\mathbb{R}^{2}\right) \times L^{2}(\mathbb{R}),
$$

where $\mathcal{D}$ is the domain of $\tilde{P}(\alpha)$.

LEMMA 4.3. For $\alpha$ large enough, the operator $\mathcal{P}(z)$ is uniformly invertible for $z \in \Omega_{\alpha}$. The inverse of $\mathcal{P}(z)$ is holomorphic in $z$ and given by

$$
\mathcal{E}(z)=\left(\begin{array}{cc}
E(z) & E_{+}(z) \\
E_{-}(z) & E_{-+}(z)
\end{array}\right)
$$

where

$$
E_{-+}(z)=z-x^{2}-R_{+} V^{w}(\alpha) R_{-}-R_{+} a(z)\left[V^{w}(\alpha), \Pi\right] R_{-},
$$

with $a(z)=\left(I+\left[\Pi, V^{w}(\alpha)\right] R(z)\right)^{-1}$. Moreover,

$$
\begin{aligned}
& E(z)=R(z) a(z) ; E_{-}(z)=R_{+} a(z), \\
& E_{+}(z)=R_{-}-R(z) a(z)\left[V^{w}(\alpha), \Pi\right] R_{-} .
\end{aligned}
$$

Here $[A, B]=A B-B A$ is the commutator of $A$ and $B$. 
Proof. We claim that $\left[V^{w}(\alpha), \Pi\right]=\mathcal{O}\left(\alpha^{-1 / 2}\right)$ in $\mathcal{L}\left(L^{2}\left(\mathbb{R}^{2}\right)\right)$. Indeed, the natural restriction of $\Pi$ on $L^{2}\left(\mathbb{R}_{y}\right)$ is the projection onto the eigenspace associated to the eigenvalue 1 of the operator $D_{y}^{2}+y^{2}: L^{2}(\mathbb{R}) \rightarrow L^{2}(\mathbb{R})$. Let $f \in C_{0}^{\infty}((0,2) ; \mathbb{R})$, $f(y)=1$ for $y$ near 1 . Then one has $\Pi=f\left(D_{y}^{2}+y^{2}\right)$.

According to [12, Theorem 8.7], $\Pi$ is a pseudodifferential operator whose symbol belongs to $S^{0}\left(\langle y\rangle^{-\infty}\langle\eta\rangle^{-\infty}, \mathbb{R}^{4}\right)$. By using the pseudodifferential calculus, it follows that $\left[V^{w}(\alpha), \Pi\right]$ has an asymptotic expansion of the form

$$
\left[V^{w}(\alpha), \Pi\right]=\sum_{j=1}^{N} a_{j}^{w}\left(\frac{b}{\alpha} x, \frac{1}{\alpha} D_{x}\right) b_{j}^{w}\left(y, D_{y}\right) \alpha^{-\frac{j}{2}}+\mathcal{O}\left(\alpha^{-\frac{N+1}{2}}\right), \forall N \in \mathbb{N},
$$

where $a_{j}, b_{j} \in S^{0}\left(\mathbb{R}^{2}\right)$. Consequently, one has

$$
\left[V^{w}(\alpha), \Pi\right]=\mathcal{O}\left(\frac{1}{\sqrt{\alpha}}\right), \text { in } \mathcal{L}\left(L^{2}\left(\mathbb{R}^{2}\right)\right),
$$

which yields the claim.

Next we construct an approximate inverse of $\mathcal{P}(z)$ as follows:

$$
\tilde{\mathcal{E}}(z)=\left(\begin{array}{cc}
R(z) & R_{-} \\
R_{+} & z-x^{2}-R_{+} V^{w}(\alpha) R_{-}
\end{array}\right) .
$$

A straightforward computation gives

$$
\mathcal{P}(z) \tilde{\mathcal{E}}(z)=\left(\begin{array}{cc}
\tilde{P}(\alpha)-z & R_{-} \\
R_{+} & 0
\end{array}\right)\left(\begin{array}{cc}
R(z) & R_{-} \\
R_{+} & z-x^{2}-R_{+} V^{w}(\alpha) R_{-}
\end{array}\right)=:\left(\begin{array}{cc}
A_{1} & A_{2} \\
A_{3} & A_{4}
\end{array}\right),
$$

with

$$
\begin{aligned}
& A_{4}=R_{+} R_{-}=I_{L^{2}(\mathbb{R})}, \\
& A_{3}=0\left(\text { here we used } R_{+}(I-\Pi)=R_{+}-R_{+} R_{-} R_{+}=0\right), \\
& A_{2}=(\tilde{P}(\alpha)-z) R_{-}+R_{-}\left(z-x^{2}-R_{+} V^{w}(\alpha) R_{-}\right), \\
& A_{1}=(\tilde{P}(\alpha)-z) R(z)+R_{-} R_{+} .
\end{aligned}
$$

Using the fact that $\tilde{P}_{0}(\alpha)$ commutes with $\Pi$ and $\Pi R(z)=0$, one has $(\tilde{P}(\alpha)-z) R(z)=$ $I-\Pi+\Pi V^{w}(\alpha) R(z)$. Thus,

$$
A_{1}=I-\Pi+\Pi V^{w}(\alpha) R(z)+\Pi=I+\left[\Pi, V^{w}(\alpha)\right] R(z) .
$$

On the other hand, since $\left(\alpha\left(D_{y}^{2}+y^{2}\right)-\alpha\right) R_{-}=0$ and $V^{w}(\alpha) R_{-}-R_{-} R_{+} V^{w}(\alpha) R_{-}=$ $\left[V^{w}(\alpha), \Pi\right] R_{-}$, it follows that $A_{2}=\left[V^{w}(\alpha), \Pi\right] R_{-}$. Consequently,

$$
\left(\begin{array}{ll}
A_{1} & A_{2} \\
A_{3} & A_{4}
\end{array}\right)=I_{2}+\mathcal{O}\left(\frac{1}{\sqrt{\alpha}}\right)
$$

where $I_{2}$ is the identity matrix from $L^{2}\left(\mathbb{R}^{2}\right) \times L^{2}(\mathbb{R})$ to $L^{2}\left(\mathbb{R}^{2}\right) \times L^{2}(\mathbb{R})$. Therefore, for $\alpha$ large enough, $\left(\begin{array}{ll}A_{1} & A_{2} \\ A_{3} & A_{4}\end{array}\right)$ is uniformly invertible for $z \in \Omega_{\alpha}$ and

$$
\mathcal{E}_{1}(z):=\left(\begin{array}{cc}
A_{1} & A_{2} \\
A_{3} & A_{4}
\end{array}\right)^{-1}=\left(\begin{array}{cc}
a(z) & -a(z)\left[V^{w}(\alpha), \Pi\right] R_{-} \\
0 & I_{L^{2}(\mathbb{R})}
\end{array}\right),
$$


where $a(z)=\left(I+\left[\Pi, V^{w}(\alpha)\right] R(z)\right)^{-1}$.

Hence, for $\alpha$ large enough, $\mathcal{P}(z)$ is uniformly invertible for $z \in \Omega_{\alpha}$ with inverse $\mathcal{E}(z)=\tilde{\mathcal{E}}(z) \mathcal{E}_{1}(z)$. Using the explicit expressions of $\tilde{\mathcal{E}}(z)$ and $\mathcal{E}_{1}(z)$, we obtain $(4.6)$. 16])

REMARK 4.4. The following standard properties are well known ( see $[8,10,13$,

i) $z \in \sigma(\tilde{P}(\alpha)) \Leftrightarrow 0 \in \sigma\left(E_{-+}(z)\right)$.

ii) $(z-\tilde{P}(\alpha))^{-1}=-E(z)+E_{+}(z)\left(E_{-+}(z)\right)^{-1} E_{-}(z)$, for $z \in \rho(\tilde{P}(\alpha))$.

iii) $\left(E_{-+}(z)\right)^{-1}=-R_{+}(z-\tilde{P}(\alpha))^{-1} R_{-}$, for $z \in \rho(\tilde{P}(\alpha))$.

iv) $\partial_{z} E_{-+}(z)=E_{-}(z) E_{+}(z)$.

We denote $h=\alpha^{-1}$, which plays the role of semiclassical parameter. From now on, we write $\tilde{P}(h)$ (resp. $\left.V^{w}\left(h^{\frac{1}{2}}\right)\right)$ instead of $\tilde{P}(\alpha)$ (resp. $\left.V^{w}(\alpha)\right)$. The symbol of $V^{w}\left(h^{\frac{1}{2}}\right)$ is denoted by $V\left(h^{\frac{1}{2}}\right)$. In the next proposition, we obtain the main properties of $E_{-+}(z)$.

Proposition 4.5. Let $\Omega$ be a bounded open set in $\mathbb{C}$ (independent of $h$ ). Then for $h \in\left(0, h_{0}\right)\left(h_{0}>0\right.$ small enough), the following assertions hold:

i)

$$
E_{-+}(z)-\left(z-x^{2}-V^{w}\left(\left(1-h^{2}\right)^{\frac{1}{2}} x, h D_{x}\right)\right) \in \operatorname{Op}_{h}^{w}\left(S^{-1}\left(\mathbb{R}^{2}\right)\right) .
$$

ii) For $\psi \in C_{0}^{\infty}(\mathbb{R})$, the symbol of the operator $\psi(x)\left(E_{-+}(z)-\left(z-x^{2}\right)\right)$ has a complete asymptotic expansion in powers of $h$ in $S^{0}\left(\mathbb{R}^{2}\right)$. More precisely, there exists $a(x, \xi, z ; h) \sim \sum_{j=0}^{\infty} a_{j}(x, \xi, z) h^{j}$ in $S^{0}\left(\mathbb{R}^{2}\right)$ such that

$$
\psi(x)\left(E_{-+}(z)-\left(z-x^{2}\right)\right)=a^{w}\left(x, h D_{x}, z ; h\right),
$$

with

$$
a_{0}(x, \xi, z)=a_{0}(x, \xi):=-\psi(x) V(x, \xi),
$$

$$
a_{1}(x, \xi, z)=a_{1}(x, \xi):=-\frac{1}{4} \psi(x)\left(\partial_{x}^{2} V(x, \xi)+\partial_{\xi}^{2} V(x, \xi)\right)+\frac{1}{2 i} \partial_{x} \psi(x) \partial_{\xi} V(x, \xi) .
$$

Proof. i) According to Lemma 4.3, one has

$$
E_{-+}(z)-\left(z-x^{2}\right)=-R_{+} V^{w}\left(h^{\frac{1}{2}}\right) R_{-}-R_{+} a(z)\left[V^{w}\left(h^{\frac{1}{2}}\right), \Pi\right] R_{-} .
$$

We first demonstrate that $E_{-+}(z)-\left(z-x^{2}\right)$ is an $h$ - pseudodifferential operator with bounded symbol. To do this we use the Beal's characterization of $h-$ pseudodifferential operators (see [12, Chapter 8] or [13, Chapter 8]).

Let $l(x, \xi)$ be a linear form on $\mathbb{R}^{2}$ and let $l^{w}\left(x, h D_{x}\right)$ be the corresponding $h-$ pseudodifferential operator. Using the fact that $R_{+}, R_{-}$commute with $l^{w}\left(x, h D_{x}\right)$ and that $V^{w}\left(h^{\frac{1}{2}}\right)$ is an $h$-pseudodifferential operator in $x$ whose symbol is bounded operator in $y$, we obtain

$$
\begin{aligned}
{\left[l^{w}\left(x, h D_{x}\right), R_{+} V^{w}\left(h^{\frac{1}{2}}\right) R_{-}\right] } & =R_{+}\left[l^{w}\left(x, h D_{x}\right), V^{w}\left(h^{\frac{1}{2}}\right)\right] R_{-} \\
& =\mathcal{O}(h),
\end{aligned}
$$


where in the last equality, we have used $\left\|R_{+}\right\|=\mathcal{O}(1)$ and $\left\|R_{-}\right\|=\mathcal{O}(1)$.

Similarly, for $N \in \mathbb{N}$ and linear forms $l_{1}(x, \xi), \ldots, l_{N}(x, \xi)$ on $\mathbb{R}^{2}$, we also obtain

$$
\left[l_{1}^{w}\left(x, h D_{x}\right),\left[\ldots\left[l_{N}^{w}\left(x, h D_{x}\right), R_{+} V^{w}\left(h^{\frac{1}{2}}\right) R_{-}\right] \ldots\right]\right]=\mathcal{O}\left(h^{N}\right) .
$$

According to [12, Proposition 8.3], $R_{+} V^{w}\left(h^{\frac{1}{2}}\right) R_{-}$is an $h$ - pseudodifferential operator with bounded symbol.

Using the same arguments, we also obtain that the operator $R_{+} a(z)\left[V^{w}\left(h^{\frac{1}{2}}\right), \Pi\right] R_{-}$is an $h-$ pseudodifferential operator with bounded symbol. Therefore, $E_{-+}(z)-\left(z-x^{2}\right)$ is an $h$ - pseudodifferential operator with bounded symbol denoted by $\tilde{a}(x, \xi, z ; h)$.

Now we will prove that $\tilde{a}(x, \xi, z ; h)$ has an asymptotic expansion like (4.34). First, we consider the operator

$$
R_{+} V^{w}\left(h^{\frac{1}{2}}\right) R_{-}: L^{2}(\mathbb{R}) \rightarrow L^{2}(\mathbb{R}) .
$$

Recall that $V\left(h^{\frac{1}{2}}\right)=V\left(h^{\frac{1}{2}} \eta+\left(1-h^{2}\right)^{\frac{1}{2}} x, h \xi+h^{\frac{1}{2}}\left(1-h^{2}\right)^{\frac{1}{2}} y\right)$. Applying Taylor's formula to $V\left(h^{\frac{1}{2}}\right)$ at $X:=\left(\left(1-h^{2}\right)^{\frac{1}{2}} x, h \xi\right)$, we get

$$
\begin{aligned}
& V\left(h^{\frac{1}{2}}\right)=V(X)+\left[\partial_{1} V(X) \eta+\partial_{2} V(X)\left(1-h^{2}\right)^{\frac{1}{2}} y\right] h^{\frac{1}{2}} \\
& +\frac{1}{2}\left[\partial_{1}^{2} V(X) \eta^{2}+\partial_{2}^{2} V(X)\left(\left(1-h^{2}\right)^{\frac{1}{2}} y\right)^{2}+2 \partial_{1,2}^{2} V(X) \eta\left(1-h^{2}\right)^{\frac{1}{2}} y\right] h+\ldots \\
& +\mathcal{O}_{N, X}\left(\left(\eta\left(1-h^{2}\right)^{\frac{1}{2}} y\right)^{N}\right) h^{\frac{N}{2}}
\end{aligned}
$$

where $\partial_{j}$ is the partial derivative with respect to the $j$ th-variable, $j=1,2$, and $\mathcal{O}_{N, X}$ depends on $N, X$.

Notice that

$$
\begin{aligned}
& R_{+}\left[\partial_{1}^{k} \partial_{2}^{l} V(X) \eta^{k} y^{l}\right]^{w}\left(x, y, D_{x}, D_{y}\right) R_{-} u(x) \\
& =\partial_{1}^{k} \partial_{2}^{l} V^{w}\left(\left(1-h^{2}\right)^{\frac{1}{2}} x, h D_{x}\right) u(x)\left\langle\left(\eta^{k} y^{l}\right)^{w}\left(y, D_{y}\right) \phi(y), \phi(y)\right\rangle .
\end{aligned}
$$

A simple computation shows that

$$
\left(\eta^{k} y^{l}\right)^{w}\left(y, D_{y}\right) \phi(y)=\frac{1}{i^{k}} \sum_{j=0}^{\min (k, l)} C_{k}^{j} \frac{1}{2^{j}} y^{l-j} \phi^{(k-j)}(y),
$$

where $\phi^{(k-j)}(y)$ is the derivative of order $k-j$ of $\phi(y)$. When $k+l$ is an odd number, it follows from Lemma 4.1 that $\left\langle\phi^{(k-j)}(y), y^{l-j} \phi(y)\right\rangle=0$ for all $0 \leq j \leq \min (k, l)$. Therefore, for $k+l$ odd,

$$
\left\langle\left(\eta^{k} y^{l}\right)^{w}\left(y, D_{y}\right) \phi(y), \phi(y)\right\rangle=0 \text {, for all } 0 \leq j \leq \min (k, l) .
$$

Since $\phi^{\prime}(y)=-y \phi(y)$, one has $(\eta y)^{w}\left(y, D_{y}\right) \phi(y)=\frac{1}{2} \phi(y)+y \phi^{\prime}(y)=\frac{1}{2} \phi(y)-y^{2} \phi(y)$. Combining this with Lemma 4.1, we obtain

$$
\left\langle(\eta y)^{w}\left(y, D_{y}\right) \phi(y), \phi(y)\right\rangle=0 .
$$

Putting together (4.21), (4.22), (4.24) and (4.25), we get $\forall N \in \mathbb{N}$,

$$
R_{+} V^{w}\left(h^{\frac{1}{2}}\right) R_{-}=\sum_{j=0}^{N} r_{j}^{w}\left(\left(1-h^{2}\right)^{\frac{1}{2}} x, h D_{x}\right) h^{j}+\mathcal{O}\left(h^{N+1}\right),
$$


where $r_{j} \in S^{0}\left(\mathbb{R}^{2}\right)$. In particular, $r_{0}\left(\left(1-h^{2}\right)^{\frac{1}{2}} x, \xi\right)=V\left(\left(1-h^{2}\right)^{\frac{1}{2}} x, \xi\right)$ and $r_{1}((1-$ $\left.\left.h^{2}\right)^{\frac{1}{2}} x, \xi\right)=\frac{1}{4}\left(\partial_{1}^{2} V\left(\left(1-h^{2}\right)^{\frac{1}{2}} x, \xi\right)+\partial_{2}^{2} V\left(\left(1-h^{2}\right)^{\frac{1}{2}} x, \xi\right)\right)$.

Next we study the operator $R_{+} a(z)\left[V^{w}\left(h^{\frac{1}{2}}\right), \Pi\right] R_{-}$. From the definition of $a(z)$ (see Lemma 4.3), one has

$$
\begin{aligned}
& R_{+} a(z)\left[V^{w}\left(h^{\frac{1}{2}}\right), \Pi\right] R_{-} \\
& =R_{+}\left(I+\left[\Pi, V^{w}\left(h^{\frac{1}{2}}\right)\right] R(z)\right)^{-1}\left[V^{w}\left(h^{\frac{1}{2}}\right), \Pi\right] R_{-} \\
& =R_{+} \sum_{j \geq 0}(-1)^{j}\left(\left[\Pi, V^{w}\left(h^{\frac{1}{2}}\right)\right] R(z)\right)^{j}\left[V^{w}\left(h^{\frac{1}{2}}\right), \Pi\right] R_{-} \\
& =R_{+}\left[V^{w}\left(h^{\frac{1}{2}}\right), \Pi\right] R_{-}+R_{+} \sum_{j \geq 1}(-1)^{j}\left(\left[\Pi, V^{w}\left(h^{\frac{1}{2}}\right)\right] R(z)\right)^{j}\left[V^{w}\left(h^{\frac{1}{2}}\right), \Pi\right] R_{-} \\
& =R_{+} \sum_{j \geq 1}(-1)^{j}\left(\left[\Pi, V^{w}\left(h^{\frac{1}{2}}\right)\right] R(z)\right)^{j}\left[V^{w}\left(h^{\frac{1}{2}}\right), \Pi\right] R_{-},
\end{aligned}
$$

where we have used $R_{+} \Pi=R_{+}$and $\Pi R_{-}=R_{-}$to deduce $R_{+}\left[V^{w}\left(h^{\frac{1}{2}}\right), \Pi\right] R_{-}=0$.

We write

$$
\begin{aligned}
R(z) & =\left((I-\Pi)\left[h^{-1}\left(D_{y}^{2}+y^{2}-1\right)+x^{2}+V^{w}\left(h^{\frac{1}{2}}\right)\right](I-\Pi)-z\right)^{-1}(I-\Pi) \\
& =h\left((I-\Pi)\left[\left(D_{y}^{2}+y^{2}-1\right)+h x^{2}+h V^{w}\left(h^{\frac{1}{2}}\right)\right](I-\Pi)-h z\right)^{-1}(I-\Pi) \\
& =h H_{1}\left[I+h(I-\Pi)\left(V^{w}\left(h^{\frac{1}{2}}\right)-z\right)(I-\Pi) H_{1}\right]^{-1}(I-\Pi) \\
& =H_{1} \sum_{j \geq 0}(-1)^{j}\left[(I-\Pi)\left(V^{w}\left(h^{\frac{1}{2}}\right)-z\right) H_{1}\right]^{j}(I-\Pi) h^{j+1},
\end{aligned}
$$

where $H_{1}=\left((I-\Pi)\left(D_{y}^{2}+y^{2}-1+h x^{2}\right)(I-\Pi)\right)^{-1}(I-\Pi)$ is a bounded operator since $(I-\Pi)\left(\left(D_{y}^{2}+y^{2}-1\right)+h x^{2}\right)(I-\Pi) \geq(I-\Pi)$. Moreover, if we consider $H_{1}$ as an operator from $L^{2}\left(\mathbb{R}_{y}\right)$ to $L^{2}\left(\mathbb{R}_{y}\right)$ and $x$ as a parameter, we have

$$
\left\|H_{1}\right\|_{\mathcal{L}\left(L^{2}\left(\mathbb{R}_{y}\right)\right)} \leq C
$$

for some constant $C$ independent of both $x$ and $h$. Since $\partial_{x} H_{1}=-H_{1}(2 h x) H_{1}$, there also exists $C_{1}>0$ such that

$$
\left\|\partial_{x} H_{1}\right\|_{\mathcal{L}\left(L^{2}\left(\mathbb{R}_{y}\right)\right)} \leq C_{1}
$$

Similarly, we can show that

$$
\left\|\partial_{x}^{\beta} H_{1}\right\|_{\mathcal{L}\left(L^{2}\left(\mathbb{R}_{y}\right)\right)} \leq C_{\beta}
$$

for all $\beta \in \mathbb{N}$ and $C_{\beta}$ is also independent of $x$. Combining this with the fact that $H_{1}$ does not depend on the dual variable $\xi$ of $x$, we conclude that $H_{1}$ is an $h$ pseudodifferential operator in the $x$-variable whose symbol is bounded operator in the $y$-variable.

Making use of the symbolic calculus of pseudodifferential operators with operatorvalued symbols (see [2]), and using (4.10), (4.29) and (4.32), we deduce that, $\forall N \in \mathbb{N}$,

$$
R_{+} a(z)\left[V^{w}\left(h^{\frac{1}{2}}\right), \Pi\right] R_{-}=\sum_{j=0}^{N} \tilde{r}_{j}^{w}\left(x, \xi, z ; h x^{2} ;\left(1-h^{2}\right)^{\frac{1}{2}} x\right) h^{\frac{j}{2}+2}+\mathcal{O}\left(h^{\frac{N+1}{2}+2}\right)
$$


where $\tilde{r}_{j} \in S^{0}\left(\mathbb{R}^{2}\right)$.

As a consequence of (4.26) and (4.33), the operator $E_{-+}(z)-\left(z-x^{2}-V^{w}((1-\right.$ $\left.\left.\left.h^{2}\right)^{\frac{1}{2}} x, h D_{x}\right)\right)$ is an $h$ - pseudodifferential operator with symbol belonging to $S^{-1}\left(\mathbb{R}^{2}\right)$.

ii) It follows from (4.26) and (4.33) that the symbol $\tilde{a}(x, \xi, z ; h)$ of $E_{-+}(z)-\left(z-x^{2}\right)$ can be written as follows:

$$
\tilde{a}(x, \xi, z ; h) \sim \sum_{j \geq 0} \tilde{a}_{j}(x, \xi, z ; h) h^{\frac{j}{2}},
$$

where $\tilde{a}_{j}(x, \xi, z ; h)$ is of the form $r_{j}\left(x, \xi, z ; h x^{2} ;\left(1-h^{2}\right)^{\frac{1}{2}} x\right)$ with $r_{j} \in S^{0}\left(\mathbb{R}^{2}\right)$. In particular, $\tilde{a}_{0}(x, \xi, z ; h)=V\left(\left(1-h^{2}\right)^{\frac{1}{2}} x, h \xi\right), \tilde{a}_{1}(x, \xi, z ; h)=0$ and $\tilde{a}_{2}(x, \xi, z ; h)=$ $\frac{1}{4}\left(\partial_{1}^{2} V\left(\left(1-h^{2}\right)^{\frac{1}{2}} x, \xi\right)+\partial_{\xi}^{2} V\left(\left(1-h^{2}\right)^{\frac{1}{2}} x, \xi\right)\right)$.

Now we prove (4.15). Let $\psi \in C_{0}^{\infty}(\mathbb{R})$, we claim that the symbol $a(x, \xi, z ; h)$ of the operator $\psi(x)\left(E_{-+}(z)-\left(z-x^{2}\right)\right)$ has an asymptotic expansion in powers of $h^{\frac{1}{2}}$ in $S^{0}\left(\mathbb{R}^{2}\right)$

$$
a(x, \xi, z ; h) \sim \sum_{j \geq 0} \tilde{\tilde{a}}_{j}(x, \xi, z) h^{\frac{j}{2}} .
$$

Indeed, let $\tilde{\psi} \in C_{0}^{\infty}(\mathbb{R})$ such that $\tilde{\psi}=1$ on the support of $\psi$. We set $\tilde{V}\left(h^{\frac{1}{2}}\right):=$ $\tilde{\psi}(x) V\left(h^{\frac{1}{2}}\right)$ and $\tilde{R}\left(z ; h^{\frac{1}{2}}\right):=\left((I-\Pi)\left[h^{-1}\left(D_{y}^{2}+y^{2}-1\right)+x^{2} \tilde{\psi}(x)+\tilde{V}^{w}\left(h^{\frac{1}{2}}\right)\right](I-\Pi)-\right.$ $z)^{-1}(I-\Pi)$. Applying the $h-$ pseudodifferential calculus, one obtains

$$
\begin{aligned}
& a^{w}\left(x, h D_{x}, z ; h\right) \\
& =\psi(x)\left(-R_{+} \tilde{V}^{w}\left(h^{\frac{1}{2}}\right) R_{-}-R_{+}\left(I+\left[\Pi, \tilde{V}^{w}\left(h^{\frac{1}{2}}\right)\right] \tilde{R}\left(z ; h^{\frac{1}{2}}\right)\right)^{-1}\left[\tilde{V}^{w}\left(h^{\frac{1}{2}}\right), \Pi\right] R_{-}\right) \\
& +\mathcal{O}\left(h^{\infty}\right) .
\end{aligned}
$$

We apply Taylor's formula to $V\left(h^{\frac{1}{2}}\right)$ at $\left(h^{\frac{1}{2}} \eta+x, h \xi+h^{\frac{1}{2}}\left(1-h^{2}\right)^{\frac{1}{2}} y\right)$ and obtain

$$
\tilde{V}\left(h^{\frac{1}{2}}\right)=\sum_{j=0}^{N} V_{j}\left(h^{\frac{1}{2}} \eta+x, h \xi+h^{\frac{1}{2}}\left(1-h^{2}\right)^{\frac{1}{2}} y\right) \psi_{j}(x) h^{2}+\mathcal{O}\left(h^{2(N+1)}\right),
$$

where $V_{j} \in S^{0}\left(\mathbb{R}^{2}\right)$ and $\psi_{j} \in C_{0}^{\infty}(\mathbb{R})$. We next use the same arguments as (4.21), (4.26) where $V\left(h^{\frac{1}{2}}\right)$ is replaced by the right hand side of (4.37). Then we obtain, for all $N \in \mathbb{N}$,

$$
-R_{+} \tilde{V}^{w}\left(h^{\frac{1}{2}}\right) R_{-}=\sum_{j=0}^{N} v_{j}^{w}\left(x, h D_{x}\right) h^{j}+\mathcal{O}\left(h^{N+1}\right),
$$

where $v_{j} \in S^{0}\left(\mathbb{R}^{2}\right)$ is independent of $h$. In particular,

(4.39) $v_{0}(x, \xi)=-\tilde{\psi}(x) V(x, \xi) ; v_{1}(x, \xi)=-\frac{1}{4}\left(\partial_{x}^{2}(\tilde{\psi}(x) V(x, \xi))+\partial_{\xi}^{2}(\tilde{\psi}(x) V(x, \xi))\right)$.

As (4.32), it is easy to see that

$$
\tilde{R}\left(z ; h^{\frac{1}{2}}\right)=\tilde{H}_{1} \sum_{j \geq 0}(-1)^{j}\left[(I-\Pi)\left(\tilde{\psi}(x) x^{2}+\tilde{V}^{w}\left(h^{\frac{1}{2}}\right)-z\right) \tilde{H}_{1}\right]^{j}(I-\Pi) h^{j+1},
$$


where $\tilde{H}_{1}=\left((I-\Pi)\left(D_{y}^{2}+y^{2}-1\right)(I-\Pi)\right)^{-1}(I-\Pi)$. The operator $\tilde{H}_{1}$ is independent of both $x$ and $h$. Then making use of the symbolic calculus of pseudodifferential operators with operator-valued symbols, we obtain for all $N \in \mathbb{N}$,

$$
\begin{aligned}
& -R_{+}\left(I+\left[\Pi, \tilde{V}^{w}\left(h^{\frac{1}{2}}\right)\right] \tilde{R}\left(z ; h^{\frac{1}{2}}\right)\right)^{-1}\left[\tilde{V}^{w}\left(h^{\frac{1}{2}}\right), \Pi\right] R_{-} \\
= & \sum_{j=0}^{N} \tilde{v}_{j}^{w}\left(x, h D_{x}, z\right) h^{\frac{j}{2}+2}+\mathcal{O}\left(h^{\frac{N+1}{2}+2}\right),
\end{aligned}
$$

where $\tilde{v}_{j} \in S^{0}\left(\mathbb{R}^{2}\right)$ is also independent of $h$. Thus, (4.35) follows from (4.36), (4.38) and (4.41).

Put $J\left(h^{\frac{1}{2}}\right):=R_{+}\left(I+\left[\Pi, \tilde{V}^{w}\left(h^{\frac{1}{2}}\right)\right] \tilde{R}\left(z ; h^{\frac{1}{2}}\right)\right)^{-1}\left[\tilde{V}^{w}\left(h^{\frac{1}{2}}\right), \Pi\right] R_{-}$. We construct the following bounded operator, $\Sigma: L^{2}\left(\mathbb{R}^{2}\right) \rightarrow L^{2}\left(\mathbb{R}^{2}\right), \Sigma u(x, y)=u(x,-y)$. Obviously, one has $\Sigma^{2}=I, \Sigma^{*}=\Sigma$. The following lemma shows that $J\left(h^{\frac{1}{2}}\right)=J\left(-h^{\frac{1}{2}}\right)$ which completes the proof of (4.15):

LEMMA 4.6. The following assertions hold:

i) $\Sigma \tilde{V}^{w}\left(h^{\frac{1}{2}}\right) \Sigma=\tilde{V}^{w}\left(-h^{\frac{1}{2}}\right)$.

ii) $[\Sigma, \Pi]=0$.

iii) $R_{+} \Sigma=R_{+}$and $\Sigma R_{-}=R_{-}$.

iv) $\Sigma \tilde{R}\left(z ; h^{\frac{1}{2}}\right) \Sigma=\tilde{R}\left(z ;-h^{\frac{1}{2}}\right)$.

Proof. i) Let $a(x, y, \xi, \eta) \in S^{0}\left(\mathbb{R}^{4}\right)$ arbitrarily. By a change of variables, one has

$$
\begin{aligned}
& \Sigma a^{w}\left(x, y, D_{x}, D_{y}\right) \Sigma u(x, y) \\
& =\frac{1}{(2 \pi)^{2}} \iiint \int e^{i\left(\left(x-x^{\prime}\right) \xi+\left(-y-y^{\prime}\right) \eta\right)} a\left(\frac{x+x^{\prime}}{2}, \frac{-y+y^{\prime}}{2}, \xi, \eta\right) u\left(x^{\prime},-y^{\prime}\right) d x^{\prime} d y^{\prime} d \xi d \eta \\
& =\frac{1}{(2 \pi)^{2}} \iiint \int e^{i\left(\left(x-x^{\prime}\right) \xi+\left(y-y^{\prime \prime}\right) \eta^{\prime}\right)} a\left(\frac{x+x^{\prime}}{2}, \frac{-y-y^{\prime \prime}}{2}, \xi,-\eta^{\prime}\right) u\left(x^{\prime}, y^{\prime \prime}\right) d x^{\prime} d y^{\prime \prime} d \xi d \eta^{\prime} \\
& =a^{w}\left(x,-y, D_{x},-D_{y}\right) u(x, y) .
\end{aligned}
$$

Applying this to $\tilde{V}\left(h^{\frac{1}{2}}\right)$, we obtain $\Sigma \tilde{V}^{w}\left(h^{\frac{1}{2}}\right) \Sigma=\tilde{V}^{w}\left(-h^{\frac{1}{2}}\right)$.

ii) By using the definition of $\Sigma$ and $\Pi$, one has

$$
\begin{aligned}
{[\Sigma, \Pi] u(x, y) } & =\Sigma \Pi u(x, y)-\Pi u(x,-y) \\
& =\int u(x, t) \phi(t) d t \phi(-y)-\int u(x,-t) \phi(t) d t \phi(y) \\
& =\int(u(x, t)-u(x,-t)) \phi(t) d t \phi(y) .
\end{aligned}
$$

However, since the integrand in the right hand side of (4.43) is an odd function with respect to $t$, we obtain $[\Sigma, \Pi] u(x, y)=0$ for all $u \in L^{2}\left(\mathbb{R}^{2}\right)$. Therefore, $[\Sigma, \Pi]=0$.

iii) The same arguments give (iii).

iv) The assertion (iv) is an easy consequence of (i) and (ii).

4.2. Trace formulae. Assume that the hypotheses in Theorem 3.1 hold and let $\underline{a}<\bar{a}<0$ such that $\operatorname{supp} f \subset(\underline{a}, \bar{a})$. Then we can find an almost analytic extension $\tilde{f}$ of $f$ satisfying $\tilde{f} \in C_{0}^{\infty}(\Omega),\left.\tilde{f}\right|_{\mathbb{R}}=f, \bar{\partial}_{z} \tilde{f}(z)=\mathcal{O}\left(|\operatorname{Im} z|^{\infty}\right)$, where $\Omega:=(\underline{a}, \bar{a})+i(-1,1)$ and $\bar{\partial}_{z}=\frac{1}{2}\left(\frac{\partial}{\partial x}+i \frac{\partial}{\partial y}\right)$ for $z=x+i y$ (see [12]). Notice that we can take $\tilde{f}$ with 
support in an arbitrarily small neighbourhood of the support of $f$. Since the potential $V$ vanishes at infinity, one has that

$$
\Sigma_{[\underline{a}, \bar{a}]}:=\left\{(x, \xi) \in \mathbb{R}^{2} \mid x^{2}+V(x, \xi) \in[\underline{a}, \bar{a}]\right\}
$$

is a compact set in $\mathbb{R}^{2}$.

Next we are going to prove that $f(\tilde{P}(h))$ is of trace class and give a priori estimate of $\operatorname{tr}(f(\tilde{P}(h)))$.

Proposition 4.7. The operator $f(\tilde{P}(h))$ is of trace class. Moreover, for all functions $\chi \in C_{0}^{\infty}\left(\mathbb{R}^{2} ; \mathbb{R}\right)$ such that $\chi=1$ near $\Sigma_{[\underline{a}, \bar{a}]}$, we have

$$
\operatorname{tr}(f(\tilde{P}(h)))=\operatorname{tr}\left(-\frac{1}{\pi} \int \bar{\partial}_{z} \tilde{f}(z)(\tilde{E}(z))^{-1} \partial_{z} \tilde{E}(z) L(d z) \chi^{w}\left(x, h D_{x}\right)\right)+\mathcal{O}\left(h^{\infty}\right) .
$$

Here, $\tilde{f}$ is an almost analytic extension of $f$ and $\tilde{E}(z)=z-x^{2} \psi(x)+\psi(x)\left(E_{-+}(z)-\right.$ $\left.\left(z-x^{2}\right)\right)$ for some $\psi \in C_{0}^{\infty}(\mathbb{R} ;[0,1])$ such that $\psi(x) \chi(x, \xi)=\chi(x, \xi), \forall(x, \xi) \in \mathbb{R}^{2}$.

Proof. Suppose that $\chi \in C_{0}^{\infty}\left(\mathbb{R}^{2} ; \mathbb{R}\right), \chi=1$ near $\Sigma_{[\underline{a}, \bar{a}]}$. Then we can choose a function $\tilde{V} \in S^{0}\left(\mathbb{R}^{2}\right)$ such that

- $\tilde{V}(x, y)=V(x, y)$ outside a small neighbourhood of $\Sigma_{[\underline{a}, \bar{a}]}$,

- $\chi=1$ on the support of $(\tilde{V}-V)$,

- $\left|z-x^{2}-\tilde{V}\left(\left(1-h^{2}\right)^{\frac{1}{2}} x, y\right)\right| \geq \varepsilon_{0}\langle x\rangle^{2}$ for all $(x, y) \in \mathbb{R}^{2}, z \in \Omega$ and $h$ small enough.

Indeed, let $U$ be a neighbourhood of $\Sigma_{[a, \bar{a}]}$ such that $\Sigma_{[\underline{a}, \bar{a}]} \subset U$ and $\chi=1$ on $U$. Now for $(x, y) \in \mathbb{R}^{2} \backslash U$, one has either $x^{2}+\underline{+}(x, y)<\underline{a}$ or $x^{2}+V(x, y)>\bar{a}$. However, since $x^{2}+V(x, y)$ is a continuous function and $\lim _{|(x, y)| \rightarrow \infty} V(x, y)=0$, one obtains $x^{2}+V(x, y)>\bar{a}$ for all $(x, y) \in \mathbb{R}^{2} \backslash U$. From this, there also exists $\varepsilon>0$ such that $x^{2}+V(x, y) \geq \bar{a}+\varepsilon$ (or in other words $\left.V(x, y) \geq \bar{a}+\varepsilon-x^{2}\right)$ for all $(x, y) \in \mathbb{R}^{2} \backslash U$.

Hence, it suffices to choose $\tilde{V}$ such that $\tilde{V}(x, y) \geq \bar{a}+\varepsilon-x^{2}$ for $(x, y) \in \Sigma_{[\underline{a}, \bar{a}]}$ and $\tilde{V}(x, y)=V(x, y)$ for $(x, y) \in \mathbb{R}^{2} \backslash U$. Then we obtain $\tilde{V}(x, y) \geq \bar{a}+\varepsilon-x^{2}$ for $(x, y) \in$ $\mathbb{R}^{2}$ and $\operatorname{supp}(V-\tilde{V}) \subset U$. In particular, $\tilde{V}\left(\left(1-h^{2}\right)^{\frac{1}{2}} x, y\right) \geq \bar{a}+\varepsilon-\left(1-h^{2}\right) x^{2}>\bar{a}+\varepsilon-x^{2}$ uniformly for $(x, y) \in \mathbb{R}^{2}$ and $h$ small. This shows that $\left|z-x^{2}-\tilde{V}\left(\left(1-h^{2}\right)^{\frac{1}{2}} x, y\right)\right| \geq \varepsilon$ for $(x, y) \in \mathbb{R}^{2}$. Combining this with the fact that $\tilde{V}$ vanishes at infinity, one obtains that there exists $\varepsilon_{0}>0$ such that $\left|z-x^{2}-\tilde{V}\left(\left(1-h^{2}\right)^{\frac{1}{2}} x, y\right)\right| \geq \varepsilon_{0}\langle x\rangle^{2}$ for $(x, y) \in \mathbb{R}^{2}$. The we have constructed the function $\tilde{V}$ satisfying the above conditions.

Set $\tilde{E}_{-+}(z)=E_{-+}(z)+V^{w}\left(\left(1-h^{2}\right)^{\frac{1}{2}} x, h D_{x}\right)-\tilde{V}^{w}\left(\left(1-h^{2}\right)^{\frac{1}{2}} x, h D_{x}\right)$. Then the principal symbol of the symbol of $\tilde{E}_{-+}(z)$ is $z-x^{2}-\tilde{V}\left(\left(1-h^{2}\right)^{\frac{1}{2}} x, \xi\right)$ satisfying $\left|z-x^{2}-\tilde{V}\left(\left(1-h^{2}\right)^{\frac{1}{2}} x, \xi\right)\right| \geq \varepsilon_{0}\langle x\rangle^{2}$ uniformly in $z \in \Omega$ and $(x, \xi) \in \mathbb{R}^{2}$. Therefore, for $h$ small enough, the symbol of $\tilde{E}_{-+}(z)$ is elliptic and then $\tilde{E}_{-+}(z)$ is invertible. Moreover, $z \mapsto \tilde{E}_{-+}(z)^{-1}$ is holomorphic on $\Omega$.

Applying the so-called Helffer-Sjötrand formula and Remark 4.4, one has

$$
\begin{aligned}
f(\tilde{P}(h)) & =-\frac{1}{\pi} \int \bar{\partial}_{z} \tilde{f}(z)(z-\tilde{P}(h))^{-1} L(d z) \\
& =-\frac{1}{\pi} \int \bar{\partial}_{z} \tilde{f}(z)\left(-E(z)+E_{+}(z)\left(E_{-+}(z)\right)^{-1} E_{-}(z)\right) L(d z) \\
& =-\frac{1}{\pi} \int \bar{\partial}_{z} \tilde{f}(z) E_{+}(z)\left(E_{-+}(z)\right)^{-1} E_{-}(z) L(d z) .
\end{aligned}
$$


Here we have employed the fact that $E(z)$ is analytic to get $-\frac{1}{\pi} \int \bar{\partial}_{z} \tilde{f}(z) E(z) L(d z)=$ 0 .

On the other hand, the following decomposition

$$
E_{-+}(z)^{-1}=\tilde{E}_{-+}(z)^{-1}+E_{-+}(z)^{-1}\left(\tilde{E}_{-+}(z)-E_{-+}(z)\right) \tilde{E}_{-+}(z)^{-1}
$$

yields

$$
\begin{aligned}
& -\frac{1}{\pi} \int \bar{\partial}_{z} \tilde{f}(z) E_{+}(z)\left(E_{-+}(z)\right)^{-1} E_{-}(z) L(d z) \\
= & -\frac{1}{\pi} \int \bar{\partial}_{z} \tilde{f}(z) E_{+}(z) \tilde{E}_{-+}(z)^{-1} E_{-}(z) L(d z) \\
& -\frac{1}{\pi} \int \bar{\partial}_{z} \tilde{f}(z) E_{+}(z) E_{-+}(z)^{-1}\left(\tilde{E}_{-+}(z)-E_{-+}(z)\right) \tilde{E}_{-+}(z)^{-1} E_{-}(z) L(d z) \\
= & -\frac{1}{\pi} \int \bar{\partial}_{z} \tilde{f}(z) E_{+}(z) E_{-+}(z)^{-1}\left(\tilde{E}_{-+}(z)-E_{-+}(z)\right) \tilde{E}_{-+}(z)^{-1} E_{-}(z) L(d z) .
\end{aligned}
$$

In the last equality, we have employed the analyticity of $E_{+}(z) \tilde{E}_{-+}(z)^{-1} E_{-}(z)$ on the support of $\tilde{f}$ to deduce $\frac{1}{\pi} \int \bar{\partial}_{z} \tilde{f}(z) E_{+}(z) \tilde{E}_{-+}(z)^{-1} E_{-}(z) L(d z)=0$.

By the construction of $\tilde{E}_{-+}(z)$, the support of symbol of $\tilde{E}_{-+}(z)-E_{-+}(z)$ is compact. Hence, $\tilde{E}_{-+}(z)-E_{-+}(z)$ is a trace operator (see $\left.[12,28]\right)$. Thus, $f(\tilde{P}(h))$ is of trace class. Furthermore, making use of the cyclicity of the trace, one has

$$
\operatorname{tr}(f(\tilde{P}(h)))=\operatorname{tr}\left(-\frac{1}{\pi} \int \bar{\partial}_{z} \tilde{f}(z) E_{-+}(z)^{-1}\left(\tilde{E}_{-+}(z)-E_{-+}(z)\right) \tilde{E}_{-+}(z)^{-1} \partial_{z} E_{-+}(z) L(d z)\right) .
$$

Now, by choosing $h$ small enough, we have $\chi=1$ near support of $V\left(\left(1-h^{2}\right)^{\frac{1}{2}} x, \xi\right)-$ $\tilde{V}\left(\left(1-h^{2}\right)^{\frac{1}{2}} x, \xi\right)$ which is the symbol of $\left(\tilde{E}_{-+}(z)-E_{-+}(z)\right)$. It follows from $[12$, Proposition 9.5] that

$$
\left\|f(\tilde{P}(h))\left(1-\chi^{w}\left(x, h D_{x}\right)\right)\right\|_{\mathrm{tr}}=\mathcal{O}\left(h^{\infty}\right) .
$$

Consequently,

$$
\begin{aligned}
\operatorname{tr}(f(\tilde{P}(h))) & =\operatorname{tr}\left(f(\tilde{P}(h)) \chi^{w}\left(x, h D_{x}\right)\right)+\mathcal{O}\left(h^{\infty}\right) \\
& =\operatorname{tr}\left(-\frac{1}{\pi} \int \bar{\partial}_{z} \tilde{f}(z) E_{-+}(z)^{-1} \partial_{z} E_{-+}(z) \chi^{w}\left(x, h D_{x}\right) L(d z)\right)+\mathcal{O}\left(h^{\infty}\right) .
\end{aligned}
$$

Let $\psi$ be in $C_{0}^{\infty}(\mathbb{R} ;[0,1])$ such that $\psi(x) \chi(x, \xi)=\chi(x, \xi)$. If we consider the function $\psi$ as a constant function with respect to $\xi$, then $\operatorname{supp}(1-\psi) \cap \operatorname{supp} \chi=\emptyset$. Let us put

$$
\tilde{E}(z)=z-x^{2} \psi(x)+\psi(x)\left(E_{-+}(z)-\left(z-x^{2}\right)\right)
$$

According to [7, Lemma 1.1], there exists $\epsilon>0$ such that

- $\tilde{E}(z)^{-1}$ exists and is holomorphic on $\{z \in \tilde{\Omega}|| \operatorname{Im} z \mid \neq 0\}$, where $\tilde{\Omega}:=(\underline{a}, \bar{a})+$ $i(-\epsilon, \epsilon)$

- $\left\|\tilde{E}(z)^{-1}\right\|=\mathcal{O}\left(|\operatorname{Im} z|^{-1}\right)$ for $z \in \tilde{\Omega},|\operatorname{Im} z| \neq 0$.

Choosing $\tilde{f}$ with support in $\tilde{\Omega}$, we get $\tilde{E}(z)^{-1}$ exists and is holomorphic on $\{z \in$ $\operatorname{supp} \tilde{f}|| \operatorname{Im} z \mid \neq 0\}$. 
It follows from the resolvent identity that

$$
\begin{aligned}
& E_{-+}(z)^{-1}-\tilde{E}(z)^{-1} \\
& =E_{-+}(z)^{-1}\left(\tilde{E}(z)-E_{-+}(z)\right) \tilde{E}(z)^{-1} \\
& =E_{-+}(z)^{-1}\left((1-\psi(x)) x^{2}+(\psi(x)-1)\left(E_{-+}(z)-\left(z-x^{2}\right)\right)\right) \tilde{E}(z)^{-1} .
\end{aligned}
$$

Following the proof of [12, Proposition 8.6], we obtain for $z \in \operatorname{supp} \tilde{f}, \operatorname{Im} z \neq 0$, that $\tilde{E}(z)^{-1}$ is an $h$-pseudodifferential operator with symbol $e(x, \xi, z ; h)$ satisfying, for all $\beta \in \mathbb{N}^{2}$,

$$
\left|\partial^{\beta} e\right| \leq C_{\beta} \max \left(1, \frac{h^{\frac{1}{2}}}{|\operatorname{Im} z|}\right)^{5}|\operatorname{Im} z|^{-|\beta|-1} .
$$

Moreover $\bar{\partial}_{z} \tilde{f}(z)=\mathcal{O}\left(|\operatorname{Im} z|^{\infty}\right)$, then we obtain the symbol of $|\operatorname{Im} z|^{-1} \bar{\partial}_{z} \tilde{f}(z) \tilde{E}(z)^{-1}$ belongs to $S^{0}\left(\mathbb{R}^{2}\right)$. Since $\operatorname{supp}(1-\psi) \cap \operatorname{supp} \chi=\emptyset$, it follows that

$\left\|\left((\psi(x)-1) x^{2}+(1-\psi(x))\left(E_{-+}(z)-\left(z-x^{2}\right)\right)\right)|\operatorname{Im} z|^{-1} \bar{\partial}_{z} \tilde{f} \tilde{E}(z)^{-1} \partial_{z} E_{-+}(z) \chi^{w}\right\|_{\operatorname{tr}}=\mathcal{O}\left(h^{\infty}\right)$.

From this and the fact that $\left\|E_{-+}(z)^{-1}\right\|=\left\|R_{+}(z-\tilde{P}(h))^{-1} R_{-}\right\|=\mathcal{O}\left(\frac{1}{|\operatorname{Im} z|}\right)$, we get

$$
\operatorname{tr}\left(-\frac{1}{\pi} \int \bar{\partial}_{z} \tilde{f}(z)\left(E_{-+}(z)^{-1}-\tilde{E}(z)^{-1}\right) \partial_{z} E_{-+}(z) \chi^{w}\left(x, h D_{x}\right) L(d z)\right)=\mathcal{O}\left(h^{\infty}\right)
$$

Thus, (4.47) and (4.49) imply

$$
\begin{aligned}
\operatorname{tr}(f(\tilde{P}(h))) & =\operatorname{tr}\left(-\frac{1}{\pi} \int \bar{\partial}_{z} \tilde{f}(z) \tilde{E}(z)^{-1} \partial_{z} E_{-+}(z) \chi^{w}\left(x, h D_{x}\right) L(d z)\right)+\mathcal{O}\left(h^{\infty}\right) \\
& =\operatorname{tr}\left(-\frac{1}{\pi} \int \bar{\partial}_{z} \tilde{f}(z) \tilde{E}(z)^{-1} \partial_{z} \tilde{E}(z) \chi^{w}\left(x, h D_{x}\right) L(d z)\right)+\mathcal{O}\left(h^{\infty}\right)
\end{aligned}
$$

In the last equality of (4.50), we have used $\partial_{z} E_{-+}(z) \chi^{w}\left(x, h D_{x}\right)=$ $\partial_{z} \tilde{E}(z) \chi^{w}\left(x, h D_{x}\right)+\mathcal{O}\left(h^{\infty}\right)$ which follows from the fact that $\psi(x) \chi(x, \xi)=1$. This ends the proof of Proposition 4.7.

By combining the arguments in the proof of Proposition 4.7 and the techniques in [12, Chapter 12] (see also [7]), one obtains the following result:

LEMMA 4.8. Under assumptions of Theorem 3.1, we have

$$
\begin{aligned}
& \operatorname{tr}\left(f(\tilde{P}(h)) \mathcal{F}_{h}^{-1} \theta(\tau-\tilde{P}(h))\right) \\
& =\operatorname{tr}\left(-\frac{1}{\pi} \int \bar{\partial}_{z} \tilde{f}(z) \mathcal{F}_{h}^{-1} \theta(\tau-z)(\tilde{E}(z))^{-1} \partial_{z} \tilde{E}(z) L(d z) \chi^{w}\right)+\mathcal{O}\left(h^{\infty}\right),
\end{aligned}
$$

where $\chi, \tilde{E}(z)$ are constructed in the proposition 4.7

We recall that $z-\psi(x)\left(x^{2}+V(x, \xi)\right)$ is the principal symbol of the symbol of $\tilde{E}(z)$. It is a linear function with respect to $z$. Furthermore, $V$ tends to zero at infinity. Then for $z$ in some compact set of $(-\infty, 0)$ and $|(x, \xi)|$ large enough, $\mid z-$ $\psi(x)\left(x^{2}+V(x, \xi)\right) \mid \geq$ const $>0$. Thus, the following proposition is a consequence of [7, Lemma 1.2] (see also [12, Chapter 8]): 
Proposition 4.9. Let $f$ be as in Proposition 4.7. Let $a_{f}(x, \xi ; h)$ be the symbol of the operator

$$
A_{f}=-\frac{1}{\pi} \int \bar{\partial}_{z} \tilde{f}(z) \tilde{E}(z)^{-1} \partial_{z} \tilde{E}(z) L(d z)
$$

Then there exists a sequence of symbols $\left(c_{j}(x, \xi)\right)_{j \in \mathbb{N}}$ in $S^{0}\left(\mathbb{R}^{2}\right)$ such that

$$
a_{f}(x, \xi ; h) \sim \sum_{j=0}^{\infty} c_{j}(x, \xi) h^{j}
$$

Moreover,

$$
\begin{aligned}
& c_{0}(x, \xi)=f\left(\psi(x)\left(x^{2}+V(x, \xi)\right)\right), \\
& c_{1}(x, \xi)=-a_{1}(x, \xi) f^{\prime}\left(\psi(x)\left(x^{2}+V(x, \xi)\right)\right) .
\end{aligned}
$$

Here $a_{1}(x, \xi)$ is given in Proposition 4.5.

5. Proofs of Theorem 3.1 and Theorem 3.2. We start by proving Theorem 3.1. It results from Propositions 4.7 and 4.9 that for all $N \in \mathbb{N}$,

$$
\operatorname{tr}(f(\tilde{P}(h)))=\sum_{j=1}^{N} \operatorname{tr}\left(c_{j}^{w}\left(x, h D_{x}\right) \chi^{w}\left(x, h D_{x}\right)\right) h^{j}+\mathcal{O}\left(h^{N+1}\right) .
$$

Since the support of $\chi$ is compact, one has (see [28, Proposition II-56] )

$$
\operatorname{tr}\left(c_{j}^{w}\left(x, h D_{x}\right) \chi^{w}\left(x, h D_{x}\right)\right)=\frac{1}{2 \pi h} \iint c_{j}(x, \xi) \chi(x, \xi) d x d \xi .
$$

Thus, for all $N \in \mathbb{N}$

$$
\operatorname{tr}(f(\tilde{P}(h)))=\sum_{j=1}^{N} A_{j}(f) h^{j}+\mathcal{O}\left(h^{N+1}\right),
$$

where $A_{j}(f)=\frac{1}{2 \pi} \iint c_{j}(x, \xi) \chi(x, \xi) d x d \xi$.

Remark that $\psi=1$ on $\operatorname{supp} \chi$, and $\chi=1$ on the set $\left\{(x, \xi) \in \mathbb{R}^{2} \mid x^{2}+V(x, \xi) \in\right.$ $\operatorname{supp} f\}$. Therefore, $c_{0}(x, \xi) \chi(x, \xi)=f\left(x^{2}+V(x, \xi)\right)$ and

$$
A_{0}(f)=\frac{1}{2 \pi} \iint f\left(x^{2}+V(x, \xi)\right) d x d \xi
$$

On the other hand $\psi^{\prime}(x) \chi(x, \xi)=0$ for all $(x, \xi) \in \mathbb{R}^{2}$. Consequently, $c_{1}(x, \xi) \chi(x, \xi)=$ $\frac{1}{4}\left(\partial_{x}^{2} V(x, \xi)+\partial_{\xi}^{2} V(x, \xi)\right) f^{\prime}\left(x^{2}+V(x, \xi)\right)$ and

$$
A_{1}(f)=\frac{1}{8 \pi} \iint\left(\partial_{x}^{2} V(x, \xi)+\partial_{\xi}^{2} V(x, \xi)\right) f^{\prime}\left(x^{2}+V(x, \xi)\right) d x d \xi
$$

Theorem 3.1 is proved.

The following corollary is a simple consequence of Theorem 3.1. We recall that $\omega=1$. 
COROLlary 5.1. Let $\lambda$ be a negative real number. Let $N_{h}(\lambda)$ be the number of eigenvalues below $\lambda$ of $\tilde{P}(h)$. Assume that the hypotheses in Theorem 3.1 hold and that

$$
\operatorname{Vol}\left(\left\{(x, y) \in \mathbb{R}^{2} \mid x^{2}+V(x, y)=\lambda\right\}\right)=0
$$

Then,

$$
\lim _{h \rightarrow 0} h N_{h}(\lambda)=M_{0}
$$

where

$$
M_{0}=\frac{1}{2 \pi} \iint_{\left\{(x, y) \in \mathbb{R}^{2} \mid x^{2}+V(x, y) \leq \lambda\right\}} d x d y
$$

Proof. It is clear that the operator $\tilde{P}(h)$ is bounded from below. Then there exists $M>0$ such that $\tilde{P}(h)>-\frac{M}{2}$. For $\varepsilon>0$ small enough such that $\lambda+\varepsilon<0$, we put $I( \pm \varepsilon)=[-M \mp \varepsilon, \lambda \pm \varepsilon]$. Let us choose $f_{ \pm \varepsilon} \in C_{0}^{\infty}(\mathbb{R} ;[0,1])$, where $f_{+\varepsilon}=1$ on $I\left(+\frac{\varepsilon}{2}\right), f_{+\varepsilon}=0$ outside $I(+\varepsilon)$ and $f_{-\varepsilon}=1$ on $I(-\varepsilon), f_{-\varepsilon}=0$ outside $I\left(-\frac{\varepsilon}{2}\right)$. It is easy to see that

$$
f_{-\varepsilon}(\tilde{P}(h)) \leq 1_{[-M, \lambda]}(\tilde{P}(h)) \leq f_{+\varepsilon}(\tilde{P}(h)) .
$$

Here $1_{[-M, \lambda]}$ is the characteristic function of the interval $[-M, \lambda]$ and $A \geq B \Leftrightarrow A-B$ is a positive operator.

From Theorem 3.1, one has

$$
\lim _{h \rightarrow 0} h \operatorname{tr}\left(f_{+\varepsilon}(\tilde{P}(h))\right)=\frac{1}{2 \pi} \iint f_{+\varepsilon}\left(x^{2}+V(x, y)\right) d x d y
$$

and

$$
\lim _{h \rightarrow 0} h \operatorname{tr}\left(f_{-\varepsilon}(\tilde{P}(h))\right)=\frac{1}{2 \pi} \iint f_{-\varepsilon}\left(x^{2}+V(x, y)\right) d x d y .
$$

Combining this with (5.9), one gets

(5.10) $\frac{1}{2 \pi} \iint f_{-\varepsilon}\left(x^{2}+V(x, y)\right) d x d y \leq \lim _{h \rightarrow 0} h N_{h}(\lambda) \leq \frac{1}{2 \pi} \iint f_{+\varepsilon}\left(x^{2}+V(x, y)\right) d x d y$.

On the other hand

$$
\begin{aligned}
& \left|\iint\left(f_{+\varepsilon}\left(x^{2}+V(x, y)\right)-1_{[-M, \lambda]}\left(x^{2}+V(x, y)\right)\right) d x d y\right| \\
& \leq \iint 1_{[\lambda, \lambda+\varepsilon]}\left(x^{2}+V(x, y)\right) d x d y+\iint 1_{[-M-\varepsilon, M]}\left(x^{2}+V(x, y)\right) d x d y \\
& =\iint 1_{[\lambda, \lambda+\varepsilon]}\left(x^{2}+V(x, y)\right) d x d y \stackrel{\varepsilon \rightarrow 0}{\longrightarrow} \operatorname{Vol}\left(\left\{(x, y) \in \mathbb{R}^{2} \mid x^{2}+V(x, y)=\lambda\right\}\right)=0 .
\end{aligned}
$$

Here we used the fact that $x^{2}+V(x, y)>M$ for all $(x, y) \in \mathbb{R}^{2}$ to deduce $\iint 1_{[-M-\varepsilon, M]}\left(x^{2}+V(x, y)\right) d x d y=0$. Similarly, one also obtains

$$
\left|\iint\left(f_{+\varepsilon}\left(x^{2}+V(x, y)\right)-1_{[-M, \lambda]}\left(x^{2}+V(x, y)\right)\right) d x d y\right| \stackrel{\varepsilon \rightarrow 0}{\longrightarrow} 0 .
$$


Now let $\varepsilon$ tend to zero in (5.10) one obtains (5.7).

REMARK 5.2. It follows from the corollary 5.1 that $N_{h}(\lambda)=\frac{1}{h}\left(M_{0}+o(1)\right)$. Naturally we want to give a more precise estimate for the term $o(1)$. The optimal estimate is actually given in Theorem 3.3 .

Now, we are going to prove Theorem 3.2. We choose $\chi$ in Lemma 4.8 such that $\left\{(x, \xi) \in \mathbb{R}^{2} \mid x^{2}+V(x, \xi) \leq \lambda+\sigma_{0}<0\right\}$ is a subset of supp $\chi$, for some $\sigma_{0}>0$, and choose $\psi \in C_{0}^{\infty}(\mathbb{R} ;[0,1]), \psi(x) \chi(x, \xi)=\chi(x, \xi)$. Then, for $\tau \leq \lambda+\sigma_{0}$, we have

$$
\psi(x)\left(x^{2}+V(x, \xi)\right)=\tau \Leftrightarrow x^{2}+V(x, \xi)=\tau .
$$

On the other hand, under the hypotheses of Theorem 3.2, there exists $0<\sigma<\sigma_{0}$, such that

$$
\nabla_{x, \xi}\left(x^{2}+V(x, \xi)\right) \neq 0 \text { for all }(x, \xi) \in\left\{(x, \xi) \in \mathbb{R}^{2} \mid \lambda-\sigma<x^{2}+V(x, \xi)<\lambda+\sigma\right\} \text {. }
$$

It follows that the principal symbol of $\tilde{E}(z), z-\psi(x)\left(x^{2}+V(x, \xi)\right)$, is strictly microhyperbolic (in the sense of [7]) in $(\lambda-\sigma, \lambda+\sigma)$. Let $f \in C_{0}^{\infty}((\lambda-\sigma, \lambda+\sigma)$; $\mathbb{R}$ ), we apply [7, Theorem 1.8] to obtain: For $M \geq 1$, uniformly in $\tau \in(\lambda-\sigma, \lambda+\sigma)$

$$
\operatorname{tr}\left(-\frac{1}{\pi} \int \bar{\partial}_{z} \tilde{f}(z) \mathcal{F}_{h}^{-1} \theta(\tau-z)(\tilde{E}(z))^{-1} \partial_{z} \tilde{E}(z) L(d z) \chi^{w}\right)=\sum_{j=0}^{M} C_{j}(\tau) h^{j-1}+\mathcal{O}\left(h^{M}\right)
$$

where $C_{j}$ is a smooth function. In particular,

$$
C_{0}(\tau)=\frac{1}{2 \pi} f(\tau) \theta(0) \int_{\left\{(x, \xi) \in \mathbb{R}^{2} \mid \psi(x)\left(x^{2}+V(x, \xi)\right)=\tau\right\}} \frac{d S_{\tau}}{\left|\nabla_{x, \xi}\left(\psi(x)\left(x^{2}+V(x, \xi)\right)\right)\right|} .
$$

Combining this with (5.11), one has

$$
C_{0}(\tau)=\frac{1}{2 \pi} f(\tau) \theta(0) \int_{\left\{(x, \xi) \in \mathbb{R}^{2} \mid x^{2}+V(x, \xi)=\tau\right\}} \frac{d S_{\tau}}{\left|\nabla_{x, \xi}\left(x^{2}+V(x, \xi)\right)\right|} .
$$

Let us denote by $\mu_{j}(h), j=1, \ldots, m_{0}$ the eigenvalues of $\tilde{P}(h)$ in $(\lambda-\sigma, \lambda+\sigma)$. Then there exists a positive constant $\varepsilon_{0}$ such that $\min _{0 \leq j \leq m_{0}}\left|\tau-\mu_{j}(h)\right| \geq \varepsilon_{0}\langle\tau\rangle$ for all $\tau \in$ $\mathbb{R} \backslash(\lambda-\sigma, \lambda+\sigma)$. From the spectral theorem and Theorem 3.1, we have

$$
\mathcal{O}\left(\frac{1}{h}\right)=\operatorname{tr}(f(\tilde{P}(h)))=\sum_{j=1}^{m_{0}} f\left(\mu_{j}(h)\right) .
$$

Since $\mathcal{F}^{-1} \theta \in \mathcal{S}(\mathbb{R})\left(\mathcal{F}^{-1}\right.$ is the classical inverse Fourier transformation), we get, for all $N \geq 1$,

$$
\begin{aligned}
\operatorname{tr}\left(f(\tilde{P}(h)) \mathcal{F}_{h}^{-1} \theta(\tau-\tilde{P}(h))\right) & =\sum_{j=1}^{m_{0}} f\left(\mu_{j}(h)\right) \frac{1}{h} \mathcal{F}^{-1} \theta\left(\frac{\tau-\mu_{j}(h)}{h}\right) \\
& =\mathcal{O}\left(\frac{1}{h^{2}}\right) \max _{0 \leq j \leq m_{0}}\left|\frac{\tau-\mu_{j}(h)}{h}\right|^{-N} \\
& =\mathcal{O}\left(h^{N-2} /\langle\tau\rangle^{N}\right)
\end{aligned}
$$


uniformly in $\tau \in \mathbb{R} \backslash(\lambda-\sigma, \lambda+\sigma)$. Therefore, it results from (4.51), (5.13) and (5.14) that, uniformly in $\tau \in \mathbb{R}$,

$$
\operatorname{tr}\left(f(\tilde{P}(h)) \mathcal{F}_{h}^{-1} \theta(\tau-\tilde{P}(h))\right)=\sum_{j=0}^{M} C_{j}(\tau) h^{j-1}+\mathcal{O}\left(h^{M} /\langle\tau\rangle^{N}\right) .
$$

This ends the proof of Theorem 3.2.

6. Proof of Theorem 3.3. In this section, we give the proof of Theorem 3.3 which is based on Theorems 3.1, 3.2 and some Tauberian arguments.

Let $\sigma$ be given in (5.12). Choosing $f_{1} \in C_{0}^{\infty}((-\infty, \lambda-\sigma / 2) ;[0,1])$ and $f_{2} \in$ $C_{0}^{\infty}((\lambda-\sigma, \lambda+\sigma) ;[0,1])$ such that $f_{1}+f_{2}=1$ on $[-M, \lambda+\sigma / 2]$, where $M>$ $\sup _{(x, y) \in \mathbb{R}^{2}}|V(x, y)|$. Let $\mu_{0}(h) \leq \mu_{1}(h) \leq \ldots$ be the eigenvalues of $\tilde{P}(h)$ in $(-\infty, 0)$. Then,

$$
N_{h}(\lambda)=\sum_{\mu_{j}(h) \leq \lambda}\left(f_{1}+f_{2}\right)\left(\mu_{j}(h)\right)=\sum_{\mu_{j}(h)} f_{1}\left(\mu_{j}(h)\right)+\sum_{\mu_{j}(h) \leq \lambda} f_{2}\left(\mu_{j}(h)\right) .
$$

It follows from Theorem 3.1 that

$$
\sum_{\mu_{j}(h)} f_{1}\left(\mu_{j}(h)\right)=\operatorname{tr}\left(f_{1}(\tilde{P}(h))\right)=\frac{1}{2 \pi h} \iint f_{1}\left(x^{2}+V(x, y)\right) d x d y+\mathcal{O}(1) .
$$

Set

$$
M(\tau, h)=\sum_{\mu_{j}(h) \leq \tau} f_{2}\left(\mu_{j}(h)\right)
$$

Replacing $\theta$ by $\theta * \theta$ if necessary, for $C>0$ large enough, we can choose a function $\theta \in C_{0}^{\infty}\left(\left(-\frac{1}{C}, \frac{1}{C}\right) ; \mathbb{R}\right)$ such that:

$$
\text { - } \theta(0)=1 \text {, }
$$

- $\mathcal{F}^{-1} \theta \geq 0$ on $\mathbb{R}$

- $\mathcal{F}^{-1} \theta \geq \varepsilon_{0}$ on $\left[-\varepsilon_{1}, \varepsilon_{1}\right]$ for some small constants $\varepsilon_{0}, \varepsilon_{1}$.

Since $M(\tau, h)$ is monotone with respect to $\tau$, we use the Tauberian arguments (see [28, Theorem V-13]) to obtain the following result:

Proposition 6.1. Uniformly in $h$ small and $\lambda \in \mathbb{R}$ one has

$$
M(\lambda, h)=\int_{-\infty}^{\lambda} \operatorname{tr}\left(f_{2}(\tilde{P}(h)) \mathcal{F}_{h}^{-1} \theta(\tau-\tilde{P}(h))\right) d \tau+\mathcal{O}(1),
$$

where $\theta$ is constructed as above.

Applying Proposition 6.1 and Theorem 3.2, we get

$$
M(\lambda, h)=h^{-1} \int_{-\infty}^{\lambda} C_{0}(\tau) d \tau+\mathcal{O}(1)
$$

where

$$
\int_{-\infty}^{\lambda} C_{0}(\tau) d \tau=\int_{\lambda-\sigma}^{\lambda} \frac{1}{2 \pi} f_{2}(\tau) d \tau \int_{\left\{(x, y) \in \mathbb{R}^{2} \mid x^{2}+V(x, y)=\tau\right\}} \frac{d S_{\tau}}{\left|\nabla_{x, y}\left(x^{2}+V(x, y)\right)\right|}
$$


The condition (5.12) allows us to apply the coarea formula (see, e.g., [28, Lemma $\mathrm{V}-9]$ )

$$
\begin{aligned}
& f_{2}(\tau) \int_{\left\{(x, y) \in \mathbb{R}^{2} \mid x^{2}+V(x, y)=\tau\right\}} \frac{d S_{\tau}}{\left|\nabla_{x, y}\left(x^{2}+V(x, y)\right)\right|} \\
& =\partial_{\tau}\left(\iint_{\left\{(x, y) \in \mathbb{R}^{2} \mid x^{2}+V(x, y) \leq \tau\right\}} f_{2}\left(x^{2}+V(x, y)\right) d x d y\right), \forall \tau \in(\lambda-\sigma, \lambda) .
\end{aligned}
$$

Thus,

$$
\int_{-\infty}^{\lambda} C_{0}(\tau) d \tau=\frac{1}{2 \pi} \iint_{\left\{(x, y) \in \mathbb{R}^{2} \mid x^{2}+V(x, y) \leq \lambda\right\}} f_{2}\left(x^{2}+V(x, y)\right) d x d y .
$$

Finally, one obtains from (6.2), (6.3) and (6.5)

$$
\begin{aligned}
N_{h}(\lambda) & =\frac{1}{2 \pi h} \iint_{\left\{(x, y) \in \mathbb{R}^{2} \mid x^{2}+V(x, y) \leq \lambda\right\}}\left(f_{1}+f_{2}\right)\left(x^{2}+V(x, y)\right) d x d y+\mathcal{O}(1) \\
& =\frac{1}{2 \pi h} \iint_{\left\{(x, y) \in \mathbb{R}^{2} \mid x^{2}+V(x, y) \leq \lambda\right\}} d x d y+\mathcal{O}(1) .
\end{aligned}
$$

Theorem 3.3 is now proved.

A. Appendix. Let $a \in S^{0}\left(m, \mathbb{R}^{4}\right)$. Suppose that $\kappa: \mathbb{R}^{4} \rightarrow \mathbb{R}^{4}$ is a linear canonical transformation. According to [12, Theorem $A_{2}$, Chapter 7], there exists an associated unitary operator $U_{\kappa}$ such that $U_{\kappa}^{-1} a^{w} U_{\kappa}=(a \circ \kappa)^{w}$.

As mentioned in the last of section 3 , we construct some linear canonical transformations to prove the unitary equivalence of $P(b, \omega)=\left(D_{x}-b y\right)^{2}+D_{y}^{2}-\sqrt{b^{2}+\omega^{2}}+$ $\omega^{2} x^{2}+V(x, y)$ and $\tilde{P}(\alpha)=\alpha\left(D_{y}^{2}+y^{2}\right)-\alpha+x^{2}+V^{w}\left(\frac{1}{\sqrt{\alpha}} D_{y}+\frac{b}{\alpha \omega} x, \frac{\omega}{\alpha} D_{x}+\frac{b \sqrt{\alpha}}{\alpha^{2}} y\right)$,

Let $p(x, y, \xi, \eta)=(\xi-b y)^{2}+\eta^{2}-\alpha+\omega^{2} x^{2}+V(x, y)$ which is the symbol of $P(b, \omega)$, we construct the following linear canonical transformations $\kappa_{0}, \kappa_{1}, \kappa_{2}$ :

The first one is

$$
\kappa_{0}:(x, y, \xi, \eta) \mapsto\left(x+\frac{1}{b} \eta, y+\frac{1}{b} \xi, \xi, \eta\right) .
$$

Then the new symbol is given by $p_{1}(x, y, \xi, \eta)=p \circ \kappa_{0}(x, y, \xi, \eta)=(b y)^{2}+\eta^{2}-\alpha+$ $\omega^{2}\left(x+\frac{1}{b} \eta\right)^{2}+V\left(x+\frac{1}{b} \eta, y+\frac{1}{b} \xi\right)$.

The second one is given by

$$
\kappa_{1}:(x, y, \xi, \eta) \mapsto\left(x, y, \xi-\frac{\omega^{2} b}{\alpha^{2}} y, \eta-\frac{\omega^{2} b}{\alpha^{2}} x\right) .
$$

The new symbol is $p_{2}(x, y, \xi, \eta)=p_{1} \circ \kappa_{1}(x, y, \xi, \eta)=(b y)^{2}+\frac{\alpha^{2}}{b^{2}} \eta^{2}-\alpha+\frac{\omega^{2} b^{2}}{\alpha^{2}} x^{2}+$ $V\left(\frac{b^{2}}{\alpha^{2}} x+\frac{1}{b} \eta, \frac{b^{2}}{\alpha^{2}} y+\frac{1}{b} \xi\right)$.

The last one is constructed as follows

$$
\kappa_{2}:(x, y, \xi, \eta) \mapsto\left(\frac{\alpha}{\omega b} x, \frac{\sqrt{\alpha}}{b} y, \frac{\omega b}{\alpha} \xi, \frac{b}{\sqrt{\alpha}} \eta\right),
$$

then the new symbol is $\tilde{p}(x, y, \xi, \eta)=p_{2} \circ \kappa_{2}(x, y, \xi, \eta)=\alpha\left(\eta^{2}+y^{2}\right)-\alpha+x^{2}+V\left(\frac{1}{\sqrt{\alpha}} \eta+\right.$ $\left.\frac{b}{\alpha \omega} x, \frac{\omega}{\alpha} \xi+\frac{b \sqrt{\alpha}}{\alpha^{2}} y\right)$. Therefore, $P(b, \omega)=p^{w}\left(x, y, D_{x}, D_{y}\right)$ is unitarily equivalent to $\tilde{P}(\alpha)=\tilde{p}^{w}\left(x, y, D_{x}, D_{y}\right)$. 
Acknowledgements. The author would like to thank the anonymous referees for their valuable comments and suggestions to improve the quality of the paper.

\section{REFERENCES}

[1] J. Avron, I. Herbst, And B. Simon, Schrödinger operators with magnetic fields. I. General interactions, Duke Math. J., 45:4 (1978), pp. 847-883.

[2] A. Balazard-Kolein, Calcul fonctionnel pour des opérateurs $h-$ admissibles à symboles opérateurs et applications, thèse de 3ème Cycle, Univ. Nantes, 1985.

[3] V. Bruneau and M. Dimassi, Weak asymptotics of the spectral shift function, Math. Nachr., 280:11 (2007), pp. 1230-1243.

[4] V. Bruneau, P. Miranda, and G. Raikov, Discrete spectrum of quantum Hall effect Hamiltonians I. Monotone edge potentials, J. Spectr. Theory, 1:3 (2011), pp. 237-272.

[5] H. L. Cycon, R. G. Froese, W. Kirsch, and B. Simon, Schrödinger operators with application to quantum mechanics and global geometry, Texts and Monographs in Physics. Springer-Verlag, Berlin, study edition, 1987.

[6] S. De Bièvre and J. V. Pulé, Propagating edge states for a magnetic Hamiltonian, Math. Phys. Electron. J., 5:Paper 3 (1999), 17 pp. (electronic).

[7] M. Dimassi, Trace asymptotics formulas and some applications, Asymptot. Anal., 18:1-2 (1998), pp. 1-32.

[8] M. DimASSI, Développements asymptotiques de l'opérateur de Schrödinger avec champ magnétique fort, Comm. Partial Differential Equations, 26:3-4 (2001), pp. 595-627.

[9] M. Dimassi AND V. PetKov, Spectral shift function and resonances for non-semi-bounded and Stark Hamiltonians, J. Math. Pures Appl. (9), 82:10 (2003), pp. 1303-1342.

[10] M. Dimassi and V. PetKov, Resonances for magnetic Stark Hamiltonians in two-dimensional case, Int. Math. Res. Not., 77 (2004), pp. 4147-4179.

[11] M. Dimassi And V. PetKov, Spectral shift function for operators with crossed magnetic and electric fields, Rev. Math. Phys., 22:4 (2010), pp. 355-380.

[12] M. Dimassi And J. SJÖStrand, Spectral asymptotics in the semi-classical limit, volume 268 of "London Mathematical Society Lecture Note Series", Cambridge University Press, Cambridge, 1999.

[13] L. Evans And M. Zworski, Semiclassical analysis, Preprint, 2012.

[14] P. Exner, A. Joye, And H. KovaŘík, Magnetic transport in a straight parabolic channel, J. Phys. A, 34:45 (2001), pp. 9733-9752.

[15] C. Gérard, A. Martinez, and J. Sjöstrand, A mathematical approach to the effective Hamiltonian in perturbed periodic problems, Comm. Math. Phys., 142:2 (1991), pp. 217244.

[16] B. HelfFer AND J. SJÖSTRAnd, Équation de Schrödinger avec champ magnétique et équation de Harper, In "Schrödinger operators (Sønderborg, 1988)", volume 345 of "Lecture Notes in Phys.", pages 118-197. Springer, Berlin, 1989.

[17] P. D. Hislop AND I. M. Sigal, Introduction to spectral theory, volume 113 of "Applied Mathematical Sciences", Springer-Verlag, New York, 1996. With applications to Schrödinger operators.

[18] V. IVRII, Microlocal analysis and precise spectral asymptotics, Springer Monographs in Mathematics. Springer-Verlag, Berlin, 1998.

[19] F. Klopp And G. RAikov, The fate of the Landau levels under perturbations of constant sign, Int. Math. Res. Not. IMRN, 24 (2009), pp. 4726-4734.

[20] N. Macris, P. A. Martin, and J. V. Pulé, On edge states in semi-infinite quantum Hall systems, J. Phys. A, 32:10 (1999), pp. 1985-1996.

[21] H. MAтsumoto AND N. UeKI, Spectral analysis of Schrödinger operators with magnetic fields, J. Funct. Anal., 140:1 (1996), pp. 218-255.

[22] H. Matsumoto and N. UeKi, Applications of the theory of the metaplectic representation to quadratic Hamiltonians on the two-dimensional Euclidean space, J. Math. Soc. Japan, 52:2 (2000), pp. 269-292.

[23] M. Melgandd and G. Rozenblum, Eigenvalue asymptotics for weakly perturbed Dirac and Schrödinger operators with constant magnetic fields of full rank, Comm. Partial Differential Equations, 28:3-4 (2003), pp. 697-736.

[24] G. RAIKOV, Eigenvalue asymptotics for the Schrödinger operator with homogeneous magnetic potential and decreasing electric potential. I. Behaviour near the essential spectrum tips, Comm. Partial Differential Equations, 15:3 (1990), pp. 407-434.

[25] G. RAIKov, Eigenvalue asymptotics for the Schrödinger operator in strong constant magnetic 
fields, Comm. Partial Differential Equations, 23:9-10 (1998), pp. 1583-1619.

[26] G. RAIKOV AND S. WARZEL, Quasi-classical versus non-classical spectral asymptotics for magnetic Schrödinger operators with decreasing electric potentials, Rev. Math. Phys., 14:10 (2002), pp. 1051-1072.

[27] M. Reed And B. Simon, Methods of modern mathematical physics. IV. Analysis of operators, Academic Press [Harcourt Brace Jovanovich Publishers], New York, 1978.

[28] D. Robert, Autour de l'approximation semi-classique, volume 68 of Progress in Mathematics, Birkhäuser Boston Inc., Boston, MA, 1987.

[29] H. Tamura, Asymptotic distribution of eigenvalues for Schrödinger operators with homogeneous magnetic fields, Osaka J. Math., 25:3 (1988), pp. 633-647. 